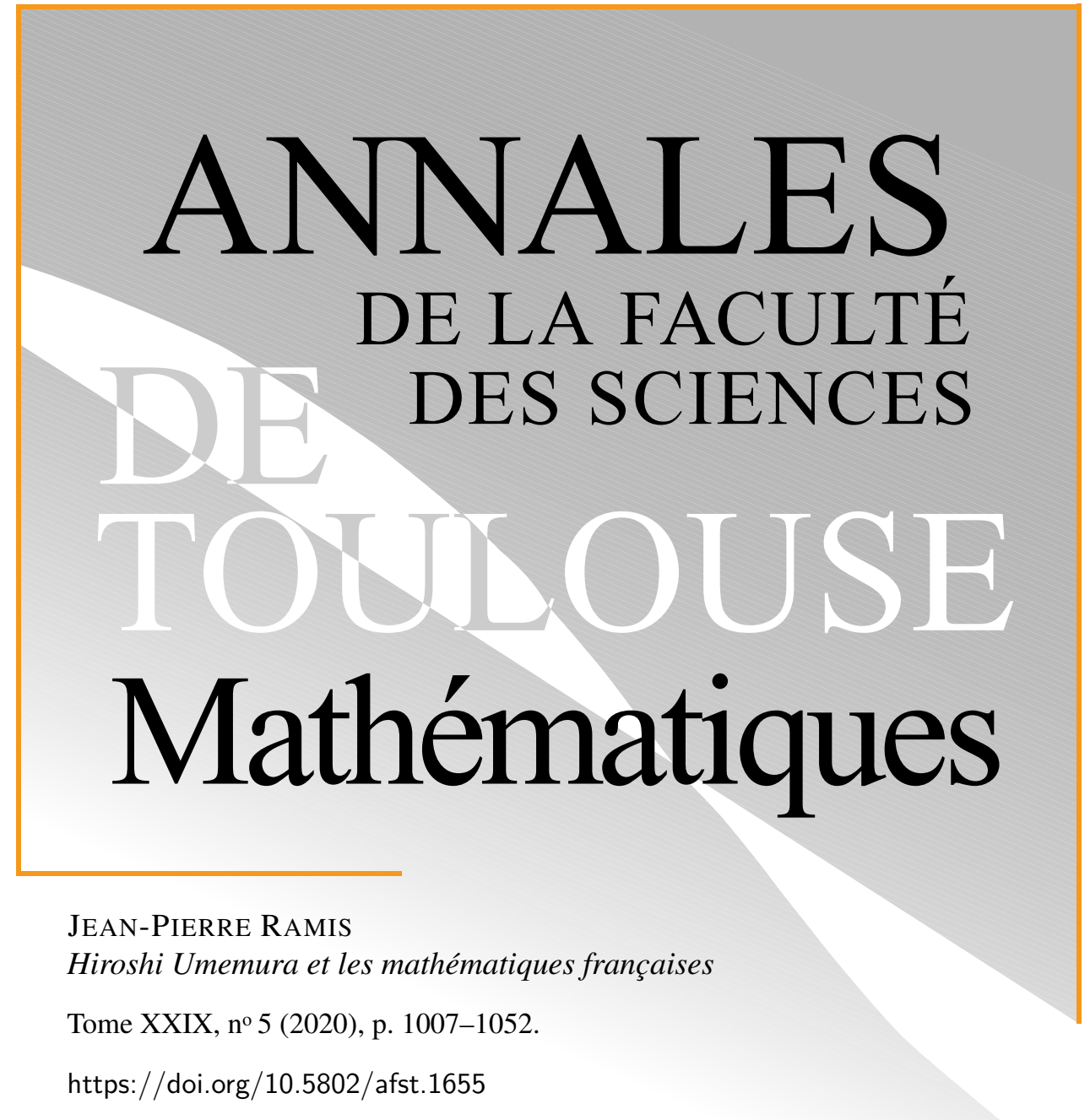

JEAN-PIERRE RAMIS

Hiroshi Umemura et les mathématiques françaises

Tome XXIX, no 5 (2020), p. 1007-1052.

https://doi.org/10.5802/afst.1655

(C) Université Paul Sabatier, Toulouse, 2020.

L'accès aux articles de la revue «Annales de la faculté des sciences de Toulouse Mathématiques » (http://afst.centre-mersenne.org/) implique l'accord avec les conditions générales d'utilisation (http://afst.centre-mersenne.org/legal/). Les articles sont publiés sous la license CC-BY 4.0.

(c) ${ }_{\mathrm{BY}}$

Publication membre du centre

Mersenne pour l'édition scientifique ouverte MERSENNE http://www.centre-mersenne.org/ 


\title{
Hiroshi Umemura et les mathématiques françaises
}

\author{
JEAN-PIERRE RAMis ${ }^{(1)}$
}

\begin{abstract}
RÉsumé. - Le but de ce texte est triple. D'abord historique : je retracerai l'évolution de deux courants mathématiques nés en France à la fin du XIX-ème siècle (l'un avec P. Painlevé, l'autre avec E. Picard, E. Vessiot et J. Drach) et leur influence sur l'oeuve de Hiroshi Umemura. Je décrirai ensuite, en entrant un peu dans la technique, le groupe de Galois de dimension infinie d'Umemura, l'une de ses contributions majeures, et ses relations avec le groupoïde que Bernard Malgrange a introduit peu après. Je livrerai aussi tout au long de ce texte un certain nombre de souvenirs personnels (comme témoin et parfois acteur). On verra apparaître, en arrière plan, un courant d'idées philosophiques auxquelles Umemura était particulièrement sensible, la théorie de l'ambiguïté, dont la pierre angulaire est la « lettre testament » d'Évariste Galois.
\end{abstract}

Abstract. - This text pursues three aims. First, a historical one : I will trace the evolution of two mathematical currents started in France at the end of the 19th century (one with P. Painlevé, the other with E. Picard, E. Vessiot et J. Drach) and their influence on the work of Hiroshi Umemura. Then, going into some technical detail, I will describe Umemura's Galois group of infinite dimension, one of his major contributions, and its relation with the groupoïd which Bernard Malgrange introduced shortly afterwards. Finally, I will give throughout the text a few personal memories (as a witness and sometimes actor). In the background, we will see emerging a current of philosophical ideas which Umemura was particularly sensitive to: the theory of ambiguity, the cornerstone of which is the "testament letter" of Evariste Galois.

Hiroshi Umemura est décédé à Nagoya le 8 mars 2019, après une terrible maladie.

Hiroshi Umemura aimait beaucoup la France, où il avait très souvent séjourné. Il a appris avec Pierre Cartier la théorie des schémas; deux séjours à Strasbourg ont joué un rôle important dans sa formation et le développement de ses idées; des courants mathématiques nés en France l'ont beaucoup influencé et il avait de forts liens d'amitié et de dialogue mathématique avec plusieurs mathématiciens français. Nous, éditeurs de ce volume, ainsi que

(1) Institut de France (Académie des Sciences) and Institut de Mathématiques de Toulouse, CNRS UMR 5219, Université Paul Sabatier (Toulouse 3), 118 route de Narbonne, 31062 Toulouse CEDEX 9, France — ramis.jean-pierre@wanadoo.fr 
Daniel Bertrand et Pierre Cartier, avons souhaité consacrer un volume spécial d'une revue française à sa mémoire. Il aimait Toulouse, avait souvent séjourné à l'Institut de Mathématiques de Toulouse et avait soumis son dernier article ${ }^{1}$ aux Annales de la Faculté des Sciences de Toulouse; ainsi le choix de cette revue était naturel et nous remercions Vincent Guedj d'avoir accueilli le volume.

Je vais retracer un certain nombre de courants scientifiques, nés en France à la fin du XIX-ème siècle qui ont beaucoup influencé H. Umemura. Il est un héritier de Painlevé, Picard, Drach et Vessiot. Les équations de Painlevé sont un thème récurrent de l'œuvre de Hiroshi. Longtemps oubliées en France elles ont été redécouvertes à Strasbourg par Georges Reeb au début des années 70. Sur cette redécouverte s'est greffée une collaboration franco-japonaise qui a joué un rôle très important pour l'évolution de la recherche sur les équations de Painlevé avec la découverte à Strasbourg par Kazuo Okamoto des variétés des conditions initiales des équations de Painlevé. Cette découverte et des travaux de l'école de Mikio Sato ont impulsé, au Japon, un grand courant de recherche sur les équations de Painlevé, dans lequel s'insère une partie de l'œuvre d'Umemura. Par ailleurs, la collaboration franco-japonaise a beaucoup contribué à l'évolution des idées d'Umemura; en particulier, pour ses travaux sur les théories de Galois en dimension infinie, qui ont beaucoup intéressé un certain nombre de mathématiciens français. Mon texte est principalement centré sur cet aspect de son œuvre ${ }^{2}$. Ses autres travaux sont analysés dans [91]. Je tenterai aussi de replacer la vision Galoisienne d'Umemura dans le grand courant des théories de l'ambiguïté ${ }^{3}$.

\section{La naissance des équations de Painlevé, leur redécouverte à Strasbourg vers 1970 et les débuts de la collaboration franco-japonaise sur les équations différentielles dans le champ complexe}

Ouverture : au commencement était Paul Painlevé ${ }^{4} .$. En m'appuyant sur l'analyse de l'œuvre scientifique de Paul Painlevé par Jacques Hada$\operatorname{mard}^{5}$ [57] (cf. aussi [94]) je vais décrire l'état de la recherche sur les équations différentielles vers 1880 et la découverte par P. Painlevé des équations qui portent son nom ${ }^{6}$.

Je cite [57] : "Pour bien comprendre non seulement le début de son œuvre ${ }^{7}$ mais le rôle qu'il joua pour toute notre génération scientifique, il faut rappeler comment, à cette époque, c'est-à-dire vers 1880-1890, évoluait la Science mathématique. Le grand problème qui s'est posé pour elle, depuis l'invention du Calcul infinitésimal, est l'intégration des équations différentielles, mais cette intégration [...] se montre, en dehors de quelques cas 
simples et vite énumérés, d'une insurmontable difficulté, qui nous apparaît de plus en plus profonde à mesure que nous connaissons mieux la nature du problème. Une grandiose découverte, qui domina toute la marche de la Science pendant toute la seconde moitié du XIX-ème siècle, permettait d'espérer à cet égard des progrès importants : ce fut la théorie des fonctions analytiques, éclairant d'une lumière nouvelle et inattendue la notion de fonction, c'està-dire la notion même du devenir. "

Hadamard parle ensuite de l'étude des singularités des équations du premier ordre $R\left(t, y, y^{\prime}\right)=0$. Dans le cas linéaire les singularités des solutions ne dépendent pas des conditions initiales, on dit que les singularités sont fixes. Mais dans le cas non-linéaire on ne peut ni prédire où apparaissent les singularités qui ne sont pas fixes, ni leur nature. Un problème naturel est de chercher les équations différentielles algébriques n'ayant ni points de ramification mobiles $^{8}$, ni singularités essentielles mobiles $^{9}$. On dit à points critiques fixes. Pour les équations du premier ordre seuls des points de ramification mobiles peuvent apparaître. L. Fuchs et H. Poincaré ont montré que les équations algébriques du premier ordre se ramènent par des transformations simples à des cas connus : linéaire, elliptique ou Riccati. La motivation initiale était de trouver des fonctions transcendantes nouvelles. En ce sens, comme l'a souligné Émile Picard, leur résultat est un échec. E. Picard (en 1893) était assez pessimiste sur la possibilité de trouver des transcendantes nouvelles en utilisant des équations d'ordre supérieur. Surmontant ce pessimisme, Painlevé a attaqué la recherche de toutes les équations à points critiques fixes pour la famille des équations différentielles rationnelles du second ordre de la forme $y^{\prime \prime}=R\left(t, y, y^{\prime}\right)$. Après une énorme quantité de calculs, il a trouvé ${ }^{10}$ que toute équation de cette forme à points critiques fixes se ramène, par des 《transformations simples », à l'un des cas déjà connus (premier ordre, linéaire, intégrable par quadratures) ou à l'un de 6 types PJ (J=I,II, . , VI). Les PJ sont les équations de Painlevé. Rappelons que le but initial était de trouver des transcendantes nouvelles, il est naturel de se demander si ce but a été atteint. Les solutions pourraient se ramener à des transcendantes « déjà connues » (et c'est le cas pour certaines valeurs des paramètres de PJ, pour $\mathrm{J} \neq \mathrm{I}$ ). C'est le problème de l'irréducibilité. Painlevé a étudié ce problème de manière approfondie depuis les Leçons de Stockholm [93].

Je cite Hadamard [57] : «On doit se demander si les équations ainsi formées ne sont réductibles par aucun moyen [...] à des équations intégrables au sens élémentaire du mot. C'est, on le sait, un genre de problèmes abordé et résolu, pour les équations algébriques, par Ruffini, Abel et Galois et auquel la science contemporaine ose s'attaquer en ce qui concerne les équations différentielles. " Hadamard explique ensuite que "les profonds travaux de 
M. Drach, posant et résolvant la question de la réductibilité des équations différentielles sous sa forme la plus générale et la plus exhaustive » ont permis à Painlevé de prouver l'irréductibilité des équations PJ. Mais cette affirmation de Hadamard était optimiste car l'on sait aujourd'hui qu'il y a des erreurs (graves $^{11}$ ) dans la thèse de Drach. Le premier à avoir relevé ces erreurs semble avoir été Vessiot [5] qui les a signalées à Painlevé (entre autres) qui a répondu : "Je viens de lire la thèse de Drach, et je suis absolument d'accord avec toi sur l'inexactitude des deux théorèmes fondamentaux et de leur démonstration. L'erreur est même tellement grosse que j'ai peine à concevoir qu'elle ait échappé à l'auteur et au jury». Ceci étant, je pense que Painlevé était convaincu que l'on pouvait rectifier les erreurs de Drach et utiliser sa théorie $^{12}$. En fait, il a fallu de longues années à Vessiot pour mettre en place une théorie consistante et ce n'est que bien plus tard que l'idée de Painlevé de prouver l'irréductibilité en utilisant une théorie de Galois différentielle non-linéaire a pu prendre corps. Il a fallu pour cela la théorie de Galois différentielle de Malgrange ; l'exemple de PI a alors pu être traité par G. Casale ${ }^{13}$ dans la ligne de Painlevé [24, 26].

Les indications qui suivent proviennent de l'introduction d'un article de G. Casale [26]. Je reviendrai plus loin sur ce sujet car Umemura a beaucoup travaillé sur ces questions et l'on peut penser que la tentative de Painlevé est ce qui l'a conduit à introduire sa théorie de Galois différentielle non linéaire (l'une de ses réussites majeures).

"Une première définition d'équation différentielle réductible a été donnée par Painlevé ${ }^{14}$ et sera ensuite formalisée ${ }^{15}$ par Nishioka [84].

Après avoir étudié l'irréductibilité des équations du premier ordre, Painlevé se pose la question de l'irréductibilité de l'équation $\frac{\mathrm{d}^{2} y}{\mathrm{~d} x^{2}}=6 y^{2}+x(\mathrm{PI})$. Il prouve dans [95] qu'au moins une solution de cette équation est irréductible puis affirme dans [96] avoir déterminé le «groupe de rationalité »de J. Drach de cette équation et prouvé ainsi son irréductibilité " absolue ». Le groupe de rationalité utilisé par P. Painlevé provient d'une tentative de J. Drach de mettre en place une théorie " de la rationalité » (ou "de Galois») valide pour toute équation différentielle [43]. Malheureusement, les travaux de J. Drach sont entachés d'erreurs. A la fin des années soixante-dix, l'école japonaise reprend et continue les travaux de Painlevé. Une preuve de l'irréductibilité de PI est enfin obtenue par K. Nishioka [84] puis par H. Umemura [U9, U10].»

Il est important, pour la compréhension de la suite de l'histoire, de souligner que, comme le dieu romain Janus, les équations de Painlevé ont deux faces. Indépendamment de Painlevé et Gambier, l'équation Painlevé VI a 
été découverte par Richard Fuchs ${ }^{16}$ par une approche complètement différente $[49,50]$ : l'étude des déformations isomonodromiques des équations linéaires fuchsiennes sur la sphère de Riemann ${ }^{17}$. En 1919, René Garnier a interprété les autres équations de Painlevé comme déformations iso-irrégulières d'équations linéaires [51]. Il ne définissait pas l'iso-irrégularité en utilisant les matrices de Stokes ${ }^{18}$, mais par un intéressant procédé de confluence de la monodromie ${ }^{19}$. Ce remarquable article de Garnier ${ }^{20}$ semble aujourd'hui oublié $^{21}$. Le résultat a été redécouvert bien plus tard par l'école japonaise [63].

La définition des équations de Painlevé par isomonodromie ou iso-irrégularité est sans doute «meilleure $»^{22}$ que la définition initiale de Painlevééc ${ }^{23}$. Je cite D. Bertrand et H. Umemura [U1] : "It is, however, very lucky that he could discover the Painlevé équations in this manner. Ceci étant, les deux approches gardent chacune leurs avantages.

Painlevé a quitté les mathématiques pour la théorie de l'aéronautique, puis pour la politique. Il a été député, puis ministre de l'instruction publique, ministre de l'air, ministre de la guerre ${ }^{24}$ et, pour une courte période, premier ministre ${ }^{25}$. C'est peut-être pour cela qu'il n'a pas fondé d'école. Par ailleurs, son oeuvre était considérée par ses contemporains comme un "point singulier» : «[...] ce propos tenu par Henri Poincaré à M. Deslandres et que j'emprunte aux souvenirs de M. Platrier ${ }^{26}$ : "Les Mathématiques constituent un continent solidement agencé, dont tous les pays sont bien reliés les uns aux autres; l'oeuvre de Paul Painlevé est une île originale et splendide dans l'océan voisin » [57, p. 303]. »

Ses équations ont été assez rapidement oubliées ${ }^{27}$.

Sautons un demi-siècle et quittons Paris pour la province. Raymond Gérard soutient sa thèse de doctorat d'État en 1968 à Strasbourg (cf. [47]). Elle était intitulée Théorie de Fuchs sur une variété analytique complexe. Le sujet lui avait été proposé par Jean Leray ${ }^{28}$.

A cette époque, on devait aussi écrire une seconde thèse, à partir de « propositions données par la faculté ». Le sujet fut proposé par Georges Reeb $^{29}$ : réexposer les leçons de Stockholm de Paul Painlevé ${ }^{30}$

Le travail de seconde thèse de Raymond se poursuivit par l'édition des oeuvres de Painlevé en collaboration avec Georges Reeb et Antoinette $\mathrm{Sec}^{31}$

A cette époque, il n'y avait pas de recherche en France sur les équations différentielles dans le champ complexe. Le sujet était considéré comme clos, sa « densité bourbachique » était nulle (ou infiniment petite). Les oeuvres de P. Painlevé, R. Garnier ${ }^{32}$, G. Darboux étaient complètement oubliées, Bourbaki admirait beaucoup Elie Cartan mais le noyau dur de son oeuvre restait 
« en dehors des écrans radars ». Les fonctions spéciales étaient considérées comme des vieilleries et n'étaient pratiquement plus enseignées ${ }^{33}$.

G. Reeb a proposé à Raymond Gérard de relire les équations de Painlevé à la lumière des feuilletages ${ }^{34}$. Ce fut un tournant essentiel et le germe d'une longue histoire qui commença par la thèse d'A. Sec, puis le travail postdoctoral de Kazuo Okamoto à Strasbourg dont les résultats ont fleuri au Japon.

Gérard a poursuivi avec talent et opiniatreté dans la voie des systèmes de Pfaff et, plus tard des équations aux dérivées partielles dans le champ complexe [54], ignorant tranquillement, comme son maître G. Reeb, le regard un peu condescendant de nombreux mathématiciens « de l'intérieur ${ }^{35}$. Quand je suis arrivé à Strasbourg, Gérard avait un poste à Metz et animait à Strasbourg un séminaire sur les systèmes de Pfaff. Vers 1973, j'ai changé de sujet de recherche ${ }^{36}$ et j'ai commencé à suivre le séminaire de Gérard. Nous discutions beaucoup ensemble, en particulier des singularités des formes différentielles analytiques dans le plan : $\omega=a \mathrm{~d} x+b \mathrm{~d} y$. Gérard avait découvert l'existence des développements asymptotiques de Poincaré $[105,119]$ et m'avait convaincu de leur intérêt ${ }^{37}$. Un jour il est arrivé au séminaire avec à la main un petit livre rouge brique qu'il venait de découvrir à la bibliothèque ${ }^{38}$ et m'a dit : ces gens là font la même chose que nous, il faut absolument entrer en contact avec eux. Les auteurs du livre étaient Masuo Hukuhara, Tosihusa Kimura et Mme Tizuko Matuda. M. Hukuhara avait, dans les années 30, décidé de travailler sur les équations différentielles dans le champ complexe après la lecture des leçons de Stokholm ${ }^{39}$ et il avait fondé une école centrée sur ce sujet ${ }^{40}$. Il avait aussi contribué à la fondation en 1958 de la revue espérantiste Funkcialaj Ekvacioj ${ }^{41}$ où ont paru de nombreux travaux de son école.

Nous avons invité T. Kimura à passer un an à Strasbourg et ce fut le début d'une riche collaboration franco-japonaise. Peu après Kazuo Okamoto, élève de T. Kimura, est venu à Strasbourg ${ }^{42}$ pendant deux années scolaires, d'octobre 1975 à juillet $1977^{43}$. Quand Kazuo est arrivé à Strasbourg R. Gérard et A. Sec avaient réalisé le programme suggéré par G. Reeb : l'interprétation (et la généralisation) d'un certain nombre de résultats de Painlevé en termes de feuilletages analytiques complexes $[52,53]$. C'est dans ce cadre qu'a travaillé Okamoto ${ }^{44}$. Son programme de travail initial était la recherche de l'espace des conditions initiales pour la première équation de Painlevé ${ }^{45}$ Il l'a mené à bien. L'exposé où il a présenté son résultat fut un grand moment du séminaire ${ }^{46}$. R. Gérard a beaucoup admiré le résultat d'Okamoto (il avait lui même essayé de résoudre le problème en essayant de faire 2 ou 3 éclatements et s'était découragé; il en fallait 8...). Ensuite, après son exposé au séminaire ${ }^{47}$, Okamoto a cherché l'espace des conditions initiales pour les 
5 autres équations de Painlevé. Il a obtenu les résultats pour tous les cas durant son séjour strasbourgeois; la face des équations de Painlevé avait été changée. Voici, en deux mots, de quoi il s'agit. L'espace des phases d'une équation de Painlevé, par exemple la première équation $\mathrm{PI}: y^{\prime \prime}=6 y^{2}+x$, est $\left(P^{1}(\mathbb{C}) \backslash \Xi\right) \times \mathbb{C}^{2}$ (où $\Xi$ est l'ensemble des points critiques fixes). On a un fibré $\left(P^{1}(\mathbb{C}) \backslash \Xi\right) \times \mathbb{C}^{2} \rightarrow P^{1}(\mathbb{C}) \backslash \Xi$. On change de base en remplaçant $P^{1}(\mathbb{C}) \backslash \Xi$ par un revêtement universel $B$. En général, une solution a des pôles; il y a ainsi une obstruction au prolongement en temps complexe de la solution. Le feuilletage associé à l'équation n'est pas uniforme. On voudrait y remédier en remplaçant l'espace des phases naïf par $E$, l'espace total d'un fibré $(E, \pi, B)$ où l'on a remplacé chaque fibre $\mathbb{C}^{2}$ par une semi-compactification convenable ${ }^{48} \pi^{-1}(x)$ (une surface algébrique affine ${ }^{49}$ ) de telle sorte que le feuilletage devienne uniforme pour la fibration (chaque feuille est transverse aux fibres et on peut relever tout chemin continu $\gamma$ de la base $B$ à partir de tout point au-dessus de son origine). C'est ce que parvint à faire Okamoto [87]. Si l'on choisit $x_{0} \in B$, alors $\pi^{-1}\left(x_{0}\right)$ est l'espace des conditions initiales ( «Okamoto space »). L'idée de sa construction est de « remplacer » le fibré naï à fibres $\mathbb{C}^{2}$ par un fibré en surfaces de Hirzebruch ${ }^{50}$, de prolonger l'équation, puis de faire un certain nombre d'éclatements (il en faut, dans tous les cas, 8).

Les variétés des conditions initiales d'Okamoto sont des variétés algébriques affines ${ }^{51}$. Elles sont analytiquement invariantes par le flot, mais leur structure algébrique se déforme. On pouvait déjà deviner que les équations de Painlevé allaient désormais être « orchestrées » par la géométrie algébrique (cf. par exemple $[106,107])$. Dans cette perspective, la vision actuelle est très bien décrite dans $[65]^{52}$. Tout ceci ${ }^{53}$ dérive de l'oeuvre pionnière d'Okamoto.

\section{Le développement de la recherche sur les équations de Painlevé au Japon après 1975}

Quittons la France pour le Japon. La recherche sur les équations de Painlevé a connu un très important développement au Japon à partir du dernier quart du XX-ème siècle. Un certain nombre de travaux de H. Umemura s'insèrent dans ce développement et cet environnement favorable. Pour aider le lecteur à mieux situer ces travaux (analysés par ailleurs dans [91]), je vais esquisser le cadre général (cf. aussi [65]).

Vers 1930-40, Hukuhara connaissait bien l'oeuvre de Painlevé (en particulier les équations de Painlevé) et l'avait diffusée au Japon. Au début des années 70 naissent indépendamment deux courants de recherches qui vont radicalement changer les choses. 
Vers 1973, Mikio Sato, à partir de la lecture d'un preprint de Tracy, Mac Coy et $\mathrm{Wu}^{54}[80,122]$ construit, en collaboration avec Miwa et Jimbo, une théorie des champs quantiques holonomes ${ }^{55}$ (Holonomic Quantum Fields). Ces auteurs montrent que les déformations isomonodromiques sont décrites par des champs quantiques holonomes [109, 110, 111]. Dans ce cadre, les équations de Painlevé sont vues comme traduisant des déformations isomonodromiques. On est du côté de R. Fuchs plutôt que de celui de Painlevé.

Vers 1977, Kazuo Okamoto construit, nous l'avons vu plus haut, les variétés des conditions initiales des six équations de Painlevé et impulse au Japon un courant de recherche dans cette direction. Ici nous sommes dans la ligne « orthodoxe» de Painlevé.

Un peu plus tard (vers 1980) les deux groupes (Miwa et Jimbo d'un côté [62], Okamoto de l'autre [88, 90]) dégagent indépendamment, et en suivant des chemins différents, la très importante notion de fonction $\tau^{56}$. B. Malgrange s'est interrogé plus tard sur la double-face (encore Janus...) des fonctions $\tau^{57}[76]$.

Ensuite Miwa, Jimbo et Ueno développent une théorie des déformations iso-irrégulières (dans la ligne de résultats oubliés de R. Garnier [51]). Par ailleurs, Okamoto s'intéresse aux transformations de Bäcklund [89].

En 1981, Sato découvre la théorie grassmanienne ${ }^{58}$ des solitons [108, 112] (cf. l'article historique [86]). Cette remarquable découverte a eu un important impact. De nombreux travaux de Miwa, Jimbo et Okamoto sont liés à la hiérarchie des solitons, aux équations bilinéaires de Hirota, aux groupes de transformations.

Vers 2009, Umemura s'est intéressé à la théorie des solitons de Sato en relation avec sa théorie de Galois différentielle. Je reviendrai plus loin sur cette question.

$\mathrm{Au}$ fil du temps les différents courants ont fusionné et le Japon est aujourd'hui le centre mondial de la recherche sur les équations de Painlevé et leurs variantes ${ }^{59}$ : « l'île Painlevé », originale et splendide, dont parlait H. Poincaré, a été rattachée à l'archipel japonais.

\section{Les séjours de Hiroshi Umemura à Strasbourg. Vers la théorie de Galois différentielle générale}

Retournons à Strasbourg. H. Umemura est venu une première fois à Strasbourg en 1971 pour travailler avec Pierre Cartier. Il a ensuite suivi Cartier à l'I.H.E.S. ${ }^{60}$. Avant sa visite en France, Umemura avait entamé des recherches 
en géométrie algébrique (voir [91, 2]). Il connaissait bien les techniques classiques à l'époque et aussi les travaux plus anciens de l'école italienne, mais avec Pierre Cartier sa vision s'est complètement renouvelée : il a découvert les catégories, les schémas, la nouvelle vision de la géométrie algébrique due à Alexande Grothendieck, outils qu'il allait largement utiliser par la suite : ils lui ont, en particulier, donné le langage (qui avait manqué à J. Drach et E. Vessiot) pour construire une théorie de Galois différentielle en dimension infinie $^{61}$. J'y reviendrai plus loin.

Hiroshi Umemura est toujours resté en contact avec Pierre Cartier. Après avoir appris le décès de Hiroshi, Cartier a écrit : "Parmi mes nombreux élèves, Umemura occupait une place de choix, nourrie de nombreux souvenirs, tant en France qu'au Japon ».

H. Umemura a ensuite passé un an à Strasbourg en 1975-76, en même temps que K. Okamoto. Sa troisième visite débute en 1984.

En 1984 beaucoup de choses avaient changé à Strasbourg depuis les premières visites d'Umemura. Il y avait trois séminaires où l'on parlait d'équations différentielles ${ }^{62}$ : le séminaire de Gérard sur les systèmes de Pfaff, le mien sur les équations fonctionnelles et le séminaire trajectorien animé par Reeb et Godbillon. Les deux premiers étaient centrés sur le champ complexe et les auditeurs étaient pour la plupart communs aux deux séminaires ${ }^{63}$. Par ailleurs, le statut des équations différentielles dans le champ complexe avait bien changé en France (et ailleurs) depuis la thèse de Gérard. Pour le cas linéaire il y avait eu le livre de Pierre Deligne [40], puis les théorèmes d'indice de Malgrange [73] et, plus tard, ma version Gevrey ${ }^{64}$ [102]. Pour le cas nonlinéaire et les feuilletages complexes la recherche avait été beaucoup stimulée par des conjectures de Thom (à Dijon avec R. Moussu et R. Roussarie et leurs élèves et à Strasbourg avec J. Martinet et moi). Il y avait aussi eu la redécouverte et le prolongement des travaux de G.D. Birkhoff par W. Balser, Jurkät, Lutz, puis Y. Sibuya, B. Malgrange, J. Martinet et moi. En tout cas il y avait à Strasbourg un grand bouillonnement autour de ces thèmes.

Je cite Dominique Foata, dans sa notice sur R. Gérard [47] : "Strasbourg devient l'arrêt obligé pour tout ce qui compte dans le monde dans le domaine des équations différentielles, en particulier les spécialistes de l'école japonaise. »

En 1984, la collaboration entre Strasbourg et le Japon s'était considérablement amplifiée. Il y avait toujours à Strasbourg des collègues japonais sur des postes annuels de profeseurs invités ou en séjour post-doctoral. Ils aimaient l'Alsace (une région fortement attachée à ses traditions comme le 
Japon), les paysages des Vosges, les Winstubs, le vin d'Alsace et l'hospitalité de Raymond Gérard dans sa maison de Blancherupt, le plus petit village d'Alsace, dans la vallée de la Bruche.

Dans ce cadre, il était naturel que H. Umemura revienne à Strasbourg. Il est arrivé à l'automne 1984 pour un séjour d'un an. Ce séjour a joué un rôle essentiel dans une nouvelle orientation, particulièrement féconde, de ses recherches.

A Strasbourg, Umemura étudie en profondeur les leçons de Stockholm et commence à réfléchir au problème de l'irréductibilité. Il apprend aussi l'algèbre différentielle et la théorie de Picard-Vessiot (sous la forme due à Kolchin). La tentative de preuve "Galoisienne » de l'irréductibilité par Painlevé a dû le motiver pour travailler dans cette direction et, par ailleurs, le contexte Strasbourgeois était favorable : la théorie de Kolchin venait d'être redécouverte en France ${ }^{65}$ (en particulier par D. Bertrand [7, 8], qui m'en avait appris l'existence ${ }^{66}$ ) et l'on en parlait à mon séminaire ${ }^{67}$ et « dans les couloirs ».

Durant son séjour à Strasbourg, Umemura a beaucoup parlé avec JeanPierre Jouanolou qui avait été un élève de Grothendieck et s'intéressait aux systèmes de Pfaff algébriques [64] (Jouanolou suivait le séminaire de Gérard).

En octobre 1985 (à la fin du séjour d'Umemura), Gérard a organisé à Strasbourg un Colloque Franco-Japonais (le premier d'une série), intitulé Équations différentielles dans le champ complexe. Je n'ai malheureusement pas pu retrouver la liste des participants. Il y avait en tout cas : D. Bertrand, T. Kimura, M. Kohno, B. Malgrange, Y. Sibuya, H. Umemura, T. Ura, M. Yoshida et les auditeurs habituels, Strasbourgeois et Messins, des deux séminaires.

A ce colloque, Umemura a parlé de ses premiers résultats vers la solution du problème de l'irréductibité [U8]. Il a continué à travailler sur la question après son retour au Japon. K. Nishioka et Umemura ont résolu (indépendamment) le problème de l'irréductibilité de PI. Pour sa solution, Umemura a mis en place une stratégie basée sur ce qu'il appelle classical function (cf. [U11], [91] et [26]).

Il écrit dans [U10] : "In our papers [U8] and [U9], we introduced the permissible operations of constructing new functions from a set of known functions. » Et, plus loin :

"We proved in [U8] that these permissible operations are related with the algebraic group. However we introduced another permissible operation (P6) in [U9], which breaks the peaceful world of algebraic groups. 
(P6) Let $F\left(y, y^{\prime}\right)=0$ be an algebraic differential equation with known coefficients. Then any solution $f$ of $F\left(y, y^{\prime}\right)=0$ is a new known function. »

La deuxième démonstration d'Umemura pour le cas de PI reprend une démarche de Painlevé dans les leçons de Stockholm [U10]. Cette démonstration n'utilise pas de théorie de Galois différentielle de dimension infinie, mais, dans cet article, Umemura écrit : "Is it possible to give the third proof depending on the infinite dimensional differential Galois theory? The difficulty of establishing the infinite dimensional differential Galois theory is widely recognized. We analyzed however the works of the classical authors and believe that the third proof is in our shooting range.

Umemura était optimiste et il n'y a pas encore, pour tous les cas, une démonstration basée sur une théorie de Galois différentielle de dimension infinie $^{68}$ (j'y reviendrai à fin de mon texte). Ce qui était par contre, pour lui, « à portée de tir » était la construction elle-même d'une théorie de Galois différentielle de dimension infinie. A partir de 1995, ce sujet (avec diverses variantes) allait devenir le centre des recherches de Hiroshi.

H. Umemura a présenté sa théorie (qu'il appelle Infinitesimal Galois Theory et, plus tard, General Differential Galois Theory et aussi Differential Galois theory of infinite dimension) dans plusieurs articles [U12, U13, U14]. J'en donne très sommairement les grandes lignes en me basant sur [U14]. Le premier point (essentiel), sur lequel il insistait très souvent au début de ses exposés ${ }^{69}$, en donnant quelques exemples simples, est basé sur le retour à une lecture correcte de l'oeuvre originale d'Évariste Galois : ce que Galois appelle "groupes» ne sont pas des groupes mais des orbites, la bonne notion est celle de groupe opérant sur un ensemble. Ainsi pour généraliser le cas classique H. Umemura se base sur la notion d'espace homogène principal (ou torseur). C'est le fil rouge à suivre pour la plupart des théories de Galois. Il est intéressant de rapprocher ceci de l'interprétation que donne G.D. Birkhoff de la théorie de l'ambiguïté évoquée par E. Galois dans sa dernière lettre [46], écrite la nuit précédant le duel fatal ${ }^{70}:$ "Tu sais, mon cher Auguste [...] Mes principales méditations, depuis quelques temps, étaient dirigées sur l'application à l'analyse transcendante de la théorie de l'ambiguïté. Il s'agissait de voir a priori, dans une relation entre des quantités ou fonctions transcendantes ${ }^{71}$ quels échanges on pouvait faire, quelles quantités on pouvait substituer aux quantités données, sans que la relation put cesser d'avoir lieu. Cela fait reconnaître de suite l'impossibilité de beaucoup d'expressions que l'on pourrait chercher. Mais je n'ai pas le temps [...]». Birkhoff [11] rapproche la vision de Galois du Principe de raison suffisante, l'un des deux principes ${ }^{72}$ à la base de la philosophie de Leibniz, proposant ainsi une très large généralisation ${ }^{73}$ de la théorie de l'ambiguité suggérée 
par Galois. Il insiste sur un point important : «Unfortunately later students of the Theory of Groups have all too frequently forgotten that, philosophically speaking, the subject remains neither more nor less than the Theory of Ambiguity».

Comme la théorie de Kolchin, la théorie d'Umemura est une théorie basée sur la notion d'extension de corps différentiels ${ }^{74}$. Umemura attache un invariant (un groupe formel) à une extension de corps différentiels ${ }^{75} L / K$. Il écrit dans [U17, paragraphe 8] : "Since this is the only one existent invariant it is easy to imagine its theoretical importance ».

Umemura considère d'abord le foncteur en groupes :

$\Gamma_{n}$ : Catégorie des $\mathbb{Q}$-algèbres $\rightarrow$ Catégorie des groupes

des changements infinitésimaux de coordonnées (en dimension $n$ ).

Il considère ensuite ce qu'il appelle des foncteurs de Lie-Ritt : ce sont les sous-foncteurs en groupe de $\Gamma_{n}$ définis par des équations différentielles (cf. [U14, Definition (7.6), p. 380]).

Notons $A^{\natural}$ la $\mathbb{Q}$-algèbre sous-jacente à une $\mathbb{Q}$-algèbre differentielle $(A, \delta)$. Umemura introduit un outil fondamental, le morphisme de Taylor universel $^{76}$ :

$$
i: A \rightarrow A^{\natural}[[t]], \quad a \mapsto \sum_{n=0}^{\infty} \frac{\delta^{n} a}{n !} t^{n} .
$$

Ce morphisme d'anneaux peut être vu comme un morphisme d'anneaux différentiels :

$$
i:(A, \delta) \rightarrow\left(A^{\natural}[[t]], \mathrm{d} / \mathrm{d} t\right) .
$$

Soit $L / K$ une extension de corps différentiels telle que $L^{\natural}$ est finiment engendrée sur $K^{\natural}$. On choisit une base de transcendance $u=\left(u_{i}\right)$ de $L^{\natural} / K^{\natural}$. Les $\partial / \partial u_{i}: K^{\natural}(u) \rightarrow K^{\natural}(u)$ s'étendent en des $\partial / \partial u_{i}: L^{\natural} \rightarrow L^{\natural}$. On obtient ainsi un corps aux dérivations partielles $\left(L^{\natural}[[t]]\left[t^{-1}\right], \mathrm{d} / \mathrm{d} t, \partial / \partial u_{i}\right)$. On note $\mathcal{L}\left(\right.$ resp. $\mathcal{K}$ ) le sous-corps aux dérivations partielles de $L^{\natural}[[t]]\left[t^{-1}\right]$ engendré par $i(L)$ (resp. $i(K))$ et $L^{\natural}$. On vérifie que l'extension $\mathcal{L} / \mathcal{K}$ ne dépend pas du choix de la base de transcendance $u$.

On définit ensuite (de façon évidente, cf. [U14, p. 382]) un morphisme de Taylor universel :

$$
j:\left(L^{\natural}, \partial / \partial u_{1}, \ldots, \partial / \partial u_{n}\right) \rightarrow L^{\natural}\left[\left[w_{1}, \ldots, w_{n}\right]\right] .
$$

On en déduit un morphisme différentiel $L^{\natural}[[t]]\left[t^{-1}\right] \rightarrow L^{\natural}[[w, t]]\left[t^{-1}\right]$, puis, par restriction à la sous-algèbre $\mathcal{L}$, un morphisme différentiel :

$$
h: \mathcal{L} \rightarrow L^{\natural}[[w, t]]\left[t^{-1}\right] .
$$


Le point essentiel est ensuite de considérer l'ensemble des déformations infinitésimales de $h$ dans une $L^{\natural}$-algèbre $A$. On définit cet ensemble $\mathcal{F}_{\mathcal{L} / \mathcal{K}}(A)$ comme l'ensemble des morphimes $\mathcal{K}$-différentiels : $f: \mathcal{L} \rightarrow L^{\natural}[[w, t]]\left[t^{-1}\right]$ égaux à $h$ modulo les éléments nilpotents de $A$. Notant (Alg $\left./ L^{\natural}\right)$ la catégorie des $L^{\natural}$-algèbres, on obtient un foncteur :

$$
\mathcal{F}_{\mathcal{L} / \mathcal{K}}:\left(\mathrm{Alg} / L^{\natural}\right) \rightarrow(\text { Ens }) .
$$

Umemura montre qu'il existe un foncteur de Lie-Ritt $\mathcal{G}$ (défini sur $L^{\natural}$ ) opérant sur le foncteur $\mathcal{F}_{\mathcal{L} / \mathcal{K}}$ de telle sorte que $\left(\mathcal{G}, \mathcal{F}_{\mathcal{L} / \mathcal{K}}\right)$ soit un espace principal homogène. Ce foncteur est, par définition, le groupe de Galois infinitésimal Inf-gal $(L / K)$ associé à l'extension différentielle $L / K$.

L'exemple favori d'Umemura (cf. [U14, Example (8.1), p. 383]) est celui de l'équation différentielle $y^{\prime}=y$. On a $K=\mathbb{C}(x)$ et $L=(\mathbb{C}(x, y), \mathrm{d} / \mathrm{d} x)$, avec $\mathrm{d} y / \mathrm{d} x=y$. Le morphisme de Taylor universel est défini par $i: y \mapsto$ $y \exp t \in L^{\natural}[[t]]$. Il est facile de faire sur cet exemple les constructions cidessus et l'on montre que $\operatorname{Inf}-\operatorname{gal}(L / K)$ est le complété formel du groupe multiplicatif, c'est-à-dire $\widehat{G}_{m, L^{\natural}}$.

Plus généralement, Umemura montre que l'invariant $\operatorname{Inf-gal}(L / K)$ d'une extension fortement normale au sens de Kolchin, de groupe de Galois différentiel $G$, est le complété formel $\widehat{G}_{L^{\natural}}$.

La Figure 1 ci-dessous (la page 29 des transparents d'un exposé d'Umemura [U5] au colloque - pour le 80-ème anniversaire de B. Malgrange de Novembre 2006 à Marseille-Luminy) fournit une autre illustration de sa théorie, cette fois pour l'équation différentielle paramétrée ${ }^{77} x(\mathrm{~d} y / \mathrm{d} x)=t y$ sur le corps $K=C(x, t)$. On notera l'apparition en marge d'un renard et d'un tori, une allusion au sanctuaire Fushimi Inari, une façon pour Hiroshi de comparer le regard galoisien à la malice du renard, j'y reviendrai plus loin.

On notera que l'invariant $\operatorname{Inf}-\operatorname{gal}(L / K)$ « ne voit pas les extensions algébriques finies ».

Le groupe de Galois Inf-gal $(L / K)$ est défini sur le corps $L^{\natural}$. On aimerait évidemment le redescendre sur le corps des constantes $C_{K}$ de $K$. Umemura étudie cette question dans [U16, cf. Question 1(*), p. 329].

Il n'y a pas de correspondance de Galois pour Inf-gal $(L / K)$, toutefois, pour un corps différentiel intermédiaire $K \subset M \subset L$ on a (cf. [U14, Remarks 4.16(i)]) un morphisme canonique surjectif :

$$
\operatorname{Inf}-\operatorname{gal}(L / K) \rightarrow \operatorname{Inf}-\operatorname{gal}(M / K) .
$$




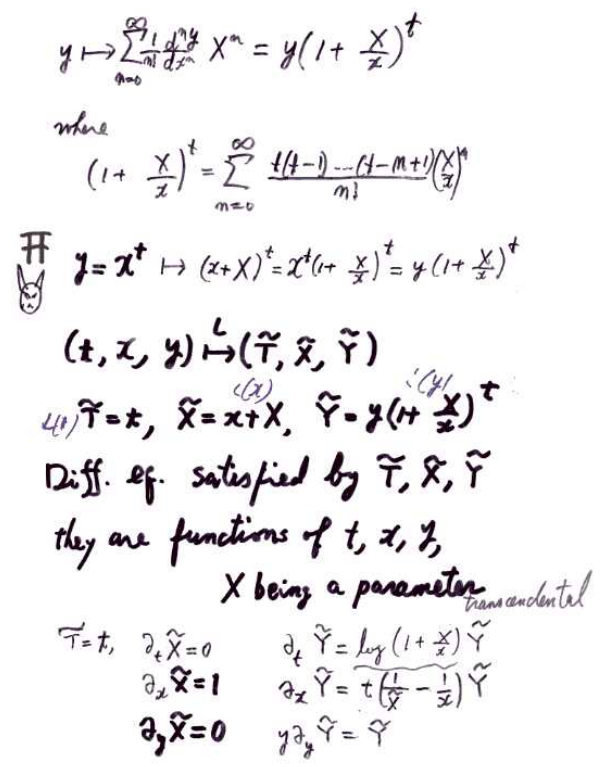

Figure 1. Morphisme de Taylor, renard et tori

Ce morphisme joue un rôle important pour les questions d'irréductibilité. C'est « un bout de correspondance de Galois ». Il n'existe apparemment rien de tel (à ma connaisance) pour la théorie de Malgrange ${ }^{78}$.

Notons pour finir que Inf-gal $(L / K)$ est un objet calculé « au point générique » et de ce fait défini sur le « gros corps $L^{\natural}$ (en similitude partielle avec le groupe de Galois intrinsèque — aussi dit : de Katz [66] — de la théorie de Picard-Vessiot, qui, lui, est défini sur le corps différentiel de base $K^{79}$ ). Umemura est revenu sur la question dans [U16] : il définit des foncteurs de Lie-Ritt sur $\mathbb{C}($ le corps des constantes de $(K, \delta)=(\mathbb{C}(x), \mathrm{d} / \mathrm{d} x))$ en utilisant des conditions initiales ${ }^{80}$. Je reviendrai plus loin sur cette question et j'expliquerai comment le morphisme de Taylor universel apparaît naturellement dans le passage au point générique.

Des analogies entre équations différentielles et équations aux différences (différences ordinaires, $q$-différences...) sont connues depuis le XVIII-éme siècle. Au XX-ème siècle ces analogies ont été développées dans diverses directions, souvent dans un esprit d'unification. Pour le point de vue de l'analyse on peut citer George D. Birkhoff [10] : "Owing to the deep-seated analogy between linear differential and difference and $q$-difference equations, [...]» et pour celui de l'algèbre Oystein Ore [92]. Pour le cas linéaire aux 
$q$-différences, on pourra consulter [42]. Des versions discrètes des équations de Painlevé sont apparues en relation avec leurs limites vers les équations de Painlevé classiques. Elles ont intéressé les physiciens dans le contexte de la gravité quantique bi-dimensionnelle ${ }^{81}$ [65]. La première construction systématique de Painlevé discrètes apparaît dans [100]. Ensuite Hidetaka Sakai a proposé une unification des équations de Painlevé classiques et des versions discrètes dans un cadre de géométrie algébrique ${ }^{82}$.

En ce qui concerne l'irréductibiité, Seiji Nishioka ${ }^{83}$ a proposé une version discrète dans [85] et a ensuite démontré l'irréductibilité de plusieurs équations de Painlevé discrètes ${ }^{84}$.

En 1963, Charles H. Franke a développé une théorie de Picard-Vessiot pour les équations aux différences, ensuite Richard P. Infante a introduit un analogue des extensions normales de Kolchin et des groupes associés. Pour le cas différentiel on travaille classiquement avec des corps différentiels ${ }^{85}$, mais si l'on procède ainsi (comme le fait Franke) pour les cas aux différences on rencontre des difficultés et il vaut mieux développer la théorie de Galois avec des anneaux aux différences simples [99]. Dans le cas non-linéaire, Anne Granier [56], Guy Casale ([23] et [31] en collaboration avec Julien Roques) ont développé des analogues discrets de la théorie de Malgrange. Pour le cas linéaire, je signale aussi deux articles plus récents $[58,41]^{86}$. Le second explore (entre autres) les relations entre le groupoïde de Malgrange et les groupes différentiels « à la Kolchin ».

En 2009 Shuji Morikawa a publié son travail de thèse sous la direction d'Umemura [82]. Il généralise la théorie d'Umemura au cas des équations « aux différences» (en particulier aux q-différences). La principale adaptation est le remplacement du morphisme de Taylor universel d'Umemura par un morphisme dit morphisme d'Euler universel (cf. [82, Definition 2.6, p. 2714 $]^{87}$ ). Morikawa et Umemura ont donné des applications de [82] à des systèmes dynamiques discrets dans [83].

En 2010, Florian Heiderich a unifié les théories d'Umemura et de Morikawa et il a levé la restriction sur la caractéristique ${ }^{88}[59,60]$. Son principal outil est l'utilisation des dérivations itérées.

Dans le dernier article ${ }^{89}$ d'Umemura, en collaboration avec A. Masuoka et K. Saito, [U2] (publié dans ce volume), les auteurs abordent le problème de l'extension des théories de Galois pour des équations fonctionnelles à un contexte permettant l'apparition de groupes quantiques comme groupes de Galois $^{90}$. Le résultat principal est la définition d'un « groupe de Galois quantique » (Algèbre de Hopf-Galois) pour des systèmes à coefficients constants. Dans la deuxième partie de l'article apparait un torseur sous un groupe quantique qui remplace les torseurs sous un groupe des cas classiques; on 
reste ainsi d'une certaine façon, dans le paradigme général. Ce travail est un article de prospective dans une direction pratiquement inexplorée et prometteuse $^{91}$. Hélas, Hiroshi nous a quittés et ne pourra pas poursuivre dans cette voie nouvelle.

Umemura parlait souvent des symétries cachées que sa théorie permet de découvrir (ou qui la fondent). Après la description de cette théorie et de ses variantes, on peut tenter une interprétation de ces symétries dans l'esprit de la théorie de l'ambiguïté (cf. la note 73). Il y a, en algèbre différentielle, des symétries abstraites, qu'un « esprit omniscient » pourrait effectivement observer. Parmi celles-ci, certaines ne sont pas observables par le pauvre algébriste différentiel, qui ne dispose que de ses outils limités : ceux-ci lui permettent seulement de voir que des contraintes algébro-différentielles ne sont pas satisfaites. Les symétries non détectables (cachées) forment un groupe ${ }^{92}$, le groupe d'Umemura ${ }^{93}$.

\section{Les théories de Galois différentielles et les contacts d'Umemura avec des mathématiciens français autour de ce thème}

Le brillant développement de la recherche japonaise sur les équations de Painlevé, que j'ai évoqué plus haut, n'a longtemps eu qu'assez peu d'échos en France, tout au moins chez les mathématiciens ${ }^{94}$. La situation pour les théories de Galois différentielles en dimension infinie est un peu inverse : les recherches d'Umemura sur ce thème me semblent avoir eu, au moins au début, un peu de difficultés à s'imposer au Japon et ont par contre rapidement intéressé plusieurs mathématiciens français. Ceci a motivé des visites régulières d'Umemura en France à partir de 2003 et plusieurs d'entre nous sont allés le voir à Nagoya. Il y a eu aussi beaucoup de rencontres à l'occasion de conférences franco-japonaises (ou à forte participation franco-japonaise) à Kyoto, Nagoya, Tokyo, Angers, Lille, Marseille-Luminy, Strasbourg, Toulouse...

Il semble que Cartier ait rencontré Umemura au Japon et l'ait invité à venir travailer avec lui à Strasbourg. Cartier a eu beaucoup d'influence sur Hiroshi, il a radicalement modifié sa vision de la géométrie algébrique. Ils se sont vus ensuite très souvent à l'occasion de divers colloques, de visites de Cartier au Japon ou des nombreux séjours d'Umemura à l'I.H.E.S. et ils ont pu confronter leurs idées, en particulier sur les théories de Galois.

La première rencontre de Daniel Bertrand avec Hiroshi date d'octobre 1985 au Colloque Franco-Japonais ${ }^{95}$, «Équations différentielles dans le 
champ complexe » à Strasbourg. Ils ont commencé à échanger sur la théorie de Galois d'Umemura une dizaine d'années plus tard. Hiroshi a invité ${ }^{96}$ Daniel à l'été $2000^{97}$. D. Bertrand et H. Umemura ont publié un article commun [U1]. Ce travail est une application de la théorie d'Umemura à la première équation de Painlevé inspirée par un article de Jules Drach [44]. Drach caractérise les solutions $\lambda(t)$ de PVI par la condition suivante : la dimension du groupe de Galois différentiel ${ }^{98}$ de l'équation différentielle non linéaire $\mathrm{d} y / \mathrm{d} t=\frac{y(y-1)(t-\lambda)}{t(t-1)(y-\lambda)}$ est finie. Daniel et Hiroshi tentent de montrer que si $\lambda(t)$ est une solution de PI et si $K=\left(\mathbb{C}\left(t, \lambda(t), \lambda^{\prime}(t)\right), L=\right.$ $K(y), y$ étant transcendant sur $K$ et solution de $\mathrm{d} y / \mathrm{d} t=\frac{1}{2} \frac{1}{y-\lambda(t)}$, alors, Inf-gal $(L / K)$ est isomorphe à $\widehat{S L}_{2, L^{\natural}}$. Umemura a précisé plus tard le résultat de Drach [U15, U16]. Il a montré ${ }^{9}$ que, dans le cadre de PVI, Inf-gal $(L / K)$ est au plus de dimension 3 .

Umemura a souvent donné des exposés à Jussieu (Paris). Bertrand et lui en profitaient pour échanger sur le groupe de Galois de Painlevé VI. Parmi les chercheurs de Jussieu avec lesquels Umemura a eu des contacts fructueux, on peut citer Florian Heiderich (invité comme post-doc à l'IMJ par Lucia Di Vizio en 2010-2011), en particulier pour les analogues de la théorie d'Umemura $[82,60]$. Pour les autres contacts de Hiroshi à Paris, citons également Michèle Loday-Richaud ${ }^{100}$ et le physicien théoricien Bernard Julia ${ }^{101}$.

Je ne sais pas où Malgrange et Umemura se sont rencontrés pour la première fois, très vraisemblablent en 1985 au Colloque Franco-Japonais à Strasbourg. Ils se sont revus plusieurs fois par la suite en France et au Japon à l'occasion de diverses conférences ${ }^{102}$. Mais ils ont évidemment eu beaucoup de discussions autour de leurs théories respectives après 2000, en particulier à l'Institut Fourier à Grenoble et à l'I.H.E.S. .

Guy Casale a soutenu une thèse ${ }^{103}$ à Toulouse en 2004, co-dirigée par Emmanuel Paul et moi. Le sujet était Sur le groupoïde de Galois d'un feuilletage [22]. Il a ensuite fait un postdoc à Tokyo ${ }^{104}$, avec Kazuo Okamoto, en 2004-2005. Durant ce séjour il a passé deux semaines à Nagoya. Il est retourné à Nagoya pour une semaine en juin 2008.

H. Umemura et Ricardo Pérez-Marco se sont rencontrés à Barcelone en 2008 à l'occasion de la première conférence $\mathrm{AMDS}^{105}$. Ils se sont revus à Tordesillas $^{106}$ à l'occasion d'une conférence en l'honneur de José-Manuel Aroca [16], où ils avaient chacun fait un exposé. Ils ont correspondu à propos des log-surfaces de Riemann de Pérez-Marco. Umemura disait que ce sujet lui rappelait ses articles des années 80. Ricardo m'a écrit : "Umemura est un de ceux qui a compris et s'est guidé par les idées transalgébriques ${ }^{107} \gg$. Le but ultime de Galois était vraisemblalement une classification des fonctions 
transcendantes « spéciales » ${ }^{108}$, Pérez-Marco et Umemura partageaient cette vision.

Après nos articles [78, 79], Jean Martinet et moi nous sommes demandés s'il y avait une théorie de Galois différentielle pouvant dire des choses sur les phénomènes de Stokes des noeuds-cols dans le plan. Nous avons essayé de comprendre la thèse de Drach, mais sans aucun succès. Ce n'est que longtemps après que Guy Casale a apporté une réponse à nos interrogations dans sa thèse [22]. Plus tard, ayant compris que le travail de Drach posait problème, j'ai plusieurs fois essayé de comprendre les articles de Vessiot, mais mes essais ont été infructueux. Ma seule contribution (au demeurant efficace) au sujet a été de parler à Malgrange du problème de définir une théorie de Galois différentielle non-linéaire, un problème qu'il ne s'était pas posé. Peu après le premier article de Malgrange sur ce sujet [74], Umemura m'a invité à Nagoya ${ }^{109}$. Nous avons évidemment parlé de sa théorie et de celle de Malgrange. J'avais des diffcultés avec la théorie de Hiroshi, il en avait avec celle de Malgrange et nous avions bien du mal à mettte les choses ensemble. Umemura m'a aussi beaucoup parlé des travaux de Vessiot. Il m'a invité deux autres fois à Nagoya ${ }^{110}$ et j'ai un souvenir très fort de la chaleureuse hospitalité de Noriko et Hiroshi. Il est venu plusieurs fois à Toulouse. Durant l'une de ses visites il devait faire une conférence sur les solitons, mais elle n'a pu avoir lieu car la ville était totalement bloquée par la neige. Nous nous sommes aussi rencontrés plusieurs fois à Kyoto. Durant la dernière de ces rencontres $^{111}$ en 2014, en pleine saison des festivités pour les momiji, nous avons longuement parlé du bouddhisme japonais (à la suite de ma visite de Kōyasan).

J'en viens maintenant à la théorie de Galois différentielle proposée par Malgrange et à la comparaison ${ }^{112}$ avec celle de Hiroshi. Je mettrai auparavant en place le cadre historique en m'appuyant sur [26] (cf. aussi $[5,18]$ ).

Je reprends, en version française, un long passage de l'introduction de [26]. Guy Casale y fait une analyse ${ }^{113}$ des relations entre les théories de Drach, Vessiot, Umemura et Malgrange.

«Entre 1887 et 1904, E. Picard [98] et E. Vessiot [116] appliquent des idées de la théorie de Galois aux équations différentielles. Ils obtiennent une théorie complète dans le cas des équations diférentielles linéaires connue aujourd'hui sous le nom de théorie de Picard-Vessiot. À peu près en même temps J. Drach [43] et E. Vessiot [117] essaient d'étendre cette théorie au cas des équations différentielles non-linéaires. Vessiot définit dans [117, 118] deux pseudogroupes réciproques ${ }^{114}$, le groupe spécifique et le groupe de rationalité $^{115}$. On trouvera cette définition ${ }^{116}$ dans l'introduction de [117, paragraphe 3]. Personne n'a suivi cette direction jusqu'à deux articles indépendants de H. Umemura [U12] et B. Malgrange [74]. Le groupe de Galois 
infinitésimal d'une équation différentielle de $H$. Umemura est le groupe de rationalité de Vessiot rigoureusement défini par un foncteur de Lie-Ritt. B. Malgrange, sans aucune connaissance de l'article tardif de Vessiot donne pratiquement la même définition que celle du groupe spécifique de Vessiot: le pseudogroupe de Galois d'un champ de vecteurs est le plus petit sous pseudogroupe algébrique contenant ce champ de vecteurs comme transformation infinitésimale.

Notons que les foncteurs de Lie-Ritt sont très voisins de la façon dont E. Cartan utilise ce que nous appelons aujoud'hui " connexions de Cartan » pour étudier les pseudogroupes de Lie. Ils sont aussi proches des "virtual groups » définis par Malgrange dans [77]. Dans le cas linéaire les deux groupes apparaissent comme groupe de Galois différentiel ${ }^{117}$ et groupe de Galois différentiel intrinsèque de N. Katz [66], qui sont respectivement le groupe de rationalité et le groupe spécifique de Vessiot. "

Je vais maintenant esquisser une description de la théorie de Galois différentielle de Bernard Malgrange. Pour alléger le texte, je me limiterai à l'idée générale $^{118}$. Les articles originaux sont $[74,75]$. Le concept de base essentiel est celui de groupoïde [21]. Un groupoïde est une petite catégorie dont toutes les flèches (ou morphismes) sont des isomorphismes.

Pour faire quelques gammes, je commence par un «baby problem»: je présente une construction du groupe de Galois différentiel d'un système différentiel rationnel sur la sphère de Riemann par adhérence de Zariski du groupoïde de monodromie. Elle est due à Cartier qui dit qu'elle est inspirée par la théorie de Malgrange ${ }^{119}$ (cf. [21, 5.2]).

Soit $(\Delta) \quad Y^{\prime}=A Y$ un système différentiel d'ordre un et de rang $n$, où $A$ est une matrice carée $(n, n)$ à coefficients rationnels. On note $S \subset \mathbb{C}$ l'ensemble des pôles de $A$ et $\Omega=\mathbb{C} \backslash S$. On choisit un point base $z_{0} \in \Omega$. A partir d'une matrice fondamentale en $z_{0}$ on associe à tout lacet continu $\gamma$ basé en $z_{0}$ sa matrice de monodromie obtenue par prolongement analytique le long de $\gamma$. On en déduit une représentation du groupe fondamental :

$$
\rho: \pi_{1}\left(\Omega, z_{0}\right) \rightarrow \mathrm{GL}(n ; \mathbb{C}),
$$

la représentation de monodromie associée au système $(\Delta)$. D'après un théorème de Schlesinger, si le système est à points singuliers réguliers $\left(\operatorname{sur} P^{1}(\mathbb{C})\right.$ ), cet invariant (transcendant) permet de récupérer le groupe de Galois différentiel du système : ce groupe (algébrique) est l'adhérence de Zariski de l'image de $\rho^{120}$ dans $\operatorname{GL}(n ; \mathbb{C})$.

C'est en général faux s'il y a au moins une singularité irrégulière. Un exemple simple ${ }^{121}$ est l'équation favorite d'Umemura $y^{\prime}=y$. L'adhérence de $\operatorname{Im} \rho$ est réduite à l'identité, mais le groupe de Galois différentiel est $\mathbb{C}^{*}$. 
Peut-on contourner la difficulté et obtenir un résultat valable dans le cas irrégulier?

On peut le faire ${ }^{122}$ en utilisant une variante du théorème de Schlesinger où l'on remplace la représentation de monodromie associée au groupe fondamental par la représentation de monodromie associée au groupoïde fondamental.

On introduit le groupoïde $\mathcal{G}$ suivant. Les objets sont les points de $\Omega$ et, pour tous $z, z^{\prime} \in \Omega$, l'ensemble des morphismes est $\operatorname{Hom}\left(z, z^{\prime}\right)=\operatorname{GL}(n ; \mathbb{C})$. On note $\left(z, z^{\prime}, g\right) \in \operatorname{Hom}\left(z, z^{\prime}\right)$. La composition est :

$$
\left(z, z^{\prime}, g\right)\left(z^{\prime}, z^{\prime \prime}, h\right)=\left(z, z^{\prime \prime}, g h\right) .
$$

On a deux applications structurales $s:\left(z, z^{\prime}, g\right) \rightarrow z$ et $b:\left(z, z^{\prime}, g\right) \rightarrow z^{\prime}$ (source et but) de $\mathcal{G}$ vers $\Omega$. On note (abusivement) $\mathcal{G}=\Omega \times \Omega \times \mathrm{GL}(n ; \mathbb{C})$.

On note $\pi_{1}(\Omega)$ le groupö̈de fondamental ${ }^{123}$ de l'espace topologique $\Omega$. Les objets sont les points de $\Omega$ et les morphismes de $z$ vers $z^{\prime}$ sont les classes d'homotopie de chemins d'origine $z$ et d'extrémité $z^{\prime}$. On définit, de manière évidente (cf. par exemple [21]) une représentation de monodromie associée au système $(\Delta): \rho: \pi_{1}(X) \rightarrow \mathcal{G}$. C'est un homomorphisme de groupoïdes.

Mais $\Omega$ et $\operatorname{GL}(n ; \mathbb{C})$ sont des variétés algébriques complexes affines. On considère l'adhérence de Zariski dans $\Omega \times \Omega \times \mathrm{GL}(n ; \mathbb{C})$ de l'image de $\rho$. C'est un sous-groupoïde $\operatorname{Gal}(\Delta)$ de $\mathcal{G}$. On peut montrer que, moyennant le choix d'un point base $z_{0} \in \Omega$, le groupe $\operatorname{Gal}(\Delta)_{z_{0}}$ associé à $\operatorname{Gal}(\Delta)$ est le groupe de Galois différentiel (au sens de Picard-Vessiot, Kolchin) du système $(\Delta)^{124}$. On peut alors dire que $\operatorname{Gal}(\Delta)$ est par définition le groupoïde de Galois différentiel de $(\Delta)$.

Revenons à l'exemple favori $y^{\prime}=y$. L'image de $\rho$ est formée des triplets $\left(z, z^{\prime}, e^{z-z^{\prime}}\right)$. L'exponentielle n'étant pas une fonction algébrique, la seule variété algébrique de $\mathbb{C} \times \mathbb{C} \times \mathbb{C}^{*}$ contenant cette image est la variété toute entière et $\operatorname{Gal}(\Delta)=\mathbb{C} \times \mathbb{C} \times \mathbb{C}^{*}$. Par suite, pour tout $z_{0} \in \mathbb{C}$, on a $\operatorname{Gal}(\Delta)=\mathbb{C}^{*}$. Comme le souligne Cartier, un point essentiel est que les opérations d'intersection avec $\left\{z=z^{\prime}=z_{0}\right\}$ et d'adhérence de Zariski ne commutent pas.

Revenons au groupoïde de Malgrange. Malgrange formule ainsi son idée de base ${ }^{125}$ : «la théorie de Galois différentielle est ce que l'algèbre voit de la dynamique ». Pour donner un sens mathématique à cette idée, il va, dans le cas d'un champ de vecteurs, utiliser une notion de clôture de Zariski du pseudogroupe engendré par le flot.

Soit $V$ une variété analytique complexe. Le point de départ de Malgrange est le groupoïde ${ }^{126} J^{*}(V)$ des germes d'applications analytiques inversibles 
de $V$ dans $V^{127}$. Il y a une variante formelle $\widehat{J}^{*}(V)$ en considérant des jets formels inversibles et des variantes $J_{k}^{*}(V)$ en considérant des jets finis inversibles d'ordre $k$. Les $J^{*}$ sont appelés groupoïdes de Lie; ils sont munis de morphismes $s$ et $b$ (source et but) vers $V$.

On suppose dans la suite que $V$ est une variété algébrique complexe (lisse séparée et connexe). Alors les $J_{k}^{*}(V)$ sont munis d'une structure de groupoïde algébrique et l'on peut voir $\widehat{J}^{*}(V)$ comme la limite projective des $J_{k}^{*}(V)$, c'est-à-dire un groupoïde pro-algébrique. Malgrange appelle sous-groupö̈de strict de $J_{k}^{*}(V)$ un sous-schéma fermé en groupoïdes.

Un $D$-groupoïde est « en gros » un sous-groupoïde de $\widehat{J}^{*}(V)$ « défini par un idéal différentiel ». Il y a plusieurs formulations rigoureuses. Dans [77, Definition 5.2] Malgrange utilise une collection $Z=\left\{Z_{k}\right\}$ de sous-schémas fermés réduits des $J_{k}^{*}(V)$ possédant un certain nombres de propriétés. Un $D$ groupoïde $Z$ a une $D$-algèbre de Lie $L Z$. On peut ensuite définir le groupoïde de Malgrange $\operatorname{Mal}(X)$ d'un champ de vecteurs rationnel $X$ sur $V$ : c'est le plus petit $D$-groupoïde $Z$ dont l'algèbre de Lie $L Z$ contient $X$ (cf. [77, Définition 5.7]). Il y a une variante pour les feuilletages. Attention : le fait qu'un tel plus petit groupoïde existe n'est pas trivial!

Pour donner une idée plus précise de cet objet sans trop entrer dans la technique, je vais utiliser la présentation de Guy Casale ${ }^{128}$ à partir de la notion d'invariant différentiel. Je suppose désormais que $V$ est affine.

Pour tout $k \in \mathbb{N}$, on définit le fibré $R_{k} V$ des $k$-repères ${ }^{129}$ (cf. [27, 2.1]). C'est une variété affine. On a une application $R_{k} V \times R_{k} V \rightarrow J_{k}^{*}(V)$ définie $\operatorname{par}(r, s) \mapsto r \circ s^{-1}$.

Les groupoïdes sont essentiellement caractérisés par leurs corps d'invariants rationnels $F_{k} \subset \mathbb{C}\left(R_{k} V\right)$ (cf. [26, Theorem 2.12]). Notons $\mathbb{C}(R V)$ la limite inductive, c'est-à-dire la réunion, des $\mathbb{C}\left(R_{k} V\right)$; c'est un corps différentiel ${ }^{130}$. Soit $F \subset \mathbb{C}(R V)$ un sous-corps différentiel. Pour tout $k$, on a un sous-corps $^{131} F_{k} \subset \mathbb{C}\left(R_{k} V\right)$.

A tout sous-corps différentiel $F \subset \mathbb{C}(R V)$ on associe un groupoïde, qui est un sous-groupoïde de $\widehat{J}^{*}(V)$ :

$$
\operatorname{Iso}(F)=\left\{\widehat{\varphi}: \widehat{(X, a)} \rightarrow \widehat{(X, a)} \mid \forall k, \forall H \in F_{k} V, H \circ R_{k} \widehat{\varphi}=H\right\} .
$$

(Si $\forall H \in F_{k} V, H \circ R_{k} \widehat{\varphi}=H$ on dit que le biholomorphisme formel $\widehat{\varphi}$ conserve $^{132} F_{k} V$.)

À un champ de vecteurs $X$ rationnel sur $V$ on associe des corps d'invariants d'ordre $k: \operatorname{Inv}_{k}(X)=\left\{H \in \mathbb{C}\left(R_{k} V\right) \mid R_{k} X . H=0\right\}$. On note $\operatorname{Inv}(X)$ leur réunion $(\operatorname{sur} k)$. Alors $\operatorname{Mal}(X)=\operatorname{Iso}(\operatorname{Inv}(X))$ (cf. [27, Definition 3.9]). 
Soient $X$ un champ de vecteurs rationnel sur $V$ et $\mathcal{F}_{X}$ le pseudo-groupe engendré par son flot, au sens ensembliste, sur les ouverts (pour la topologie ordinaire) de V. Le groupoïde de Malgrange est la clôture de Zariski de $\mathcal{F}_{X}$ dans $\widehat{J}^{*}(V)$ (cf. [27, Lemma 3.13]) : c'est bien « ce que l'algèbre voit de la dynamique $»$.

En conclusion, je signale la relation entre le troisième théorème de Lie et la théorie de Malgrange (cf. [77, Appendice B]) : "Dans la terminologie traditionnelle, le troisième théorème fondamental de Lie est l'énoncé suivant : si L est une algèbre de Lie (de dimension finie) sur $\mathbb{C}$, il existe un groupe de Lie $\mathbb{C}$-analytique d'algèbre de Lie L. Cet énoncé est faux dans le cas algébrique; l'énoncé analogue pour les pseudogroupes de Lie algébriques est également faux : ce fait est même " à la base » de la théorie de Galois différentielle telle qu'elle est présentée ici. "

H. Umemura s'est intéressé à la comparaison entre sa théorie et celle de Malgrange, dans trois articles [U16, U18, U19].

Dans [U16, 4.] il donne une version précisée de son groupe de Galois infinitésimal en prenant en compte des conditions initiales. Il se place dans un cas «modèle » qu'il note (IC) (Instructive case ${ }^{133}$ ) : $K=\mathbb{C}(x)$, $L=K\left(z_{1}, \ldots, z_{n}\right)$, les $z_{i}$ étant algébriquement dépendants sur $K, z_{i}^{\prime}=F_{i}$ (avec $\left.F_{i} \in \mathbb{C}\left[x, z_{j}\right]\right)$. C'est un système dynamique ${ }^{134}$ sur l'espace affine $\mathbb{A}^{n}$. Pour $x_{0} \in \mathbb{C}=\mathbb{A}^{1}$ fixé, les conditions initiales pour $\left(z_{1}, \ldots, z_{n}\right)$ sont $\mathbf{c}=\left(c_{1}, \ldots, c_{n}\right) \in \mathbb{A}^{n}$. On considère le pseudo-groupe de Lie $\Gamma_{n}$ des transformations birationnelles de l'espace $\mathbb{A}^{n}$ des conditions initiales.

On introduit les dérivées partielles :

$$
\partial^{m} z_{i} / \partial x^{j} \partial \mathbf{c}^{I}, \quad j \in \mathbb{N}, I \in \mathbb{N}^{n}, m=j+|I|, 1 \leqslant i \leqslant n .
$$

Ainsi $K\left(\mathbf{c}, \partial^{|I|} z_{i}, \partial \mathbf{c}^{I}\right)_{I \in \mathbb{N}^{n}}$ est un corps aux dérivées partielles. A l'extension $L / K$ on associe des contraintes sur les dérivées partielles (à coefficients dans $K(\mathbf{c})$ ) et l'on s'intéresse aux transformations $\Phi: \mathbf{c} \mapsto \Phi(\mathbf{c})$ laissant invariantes toutes les contraintes.

En fait, le corps aux dérivées partielles $K\left(\mathbf{c}, \partial^{|I|} z_{i}, \partial \mathbf{c}^{I}\right)_{I \in \mathbb{N}^{n}}$ dépend de $x_{0}$. On le note $\mathcal{L} \mid\left[x_{0}\right]$.

On fixe maintenant $\left(x_{0}, \mathbf{c}_{0}\right) \in \mathbb{A}^{1} \times \mathbb{A}^{n}$ et l'on considère le corps aux dérivations partielles $\mathbb{C}[[\underline{\mathbf{c}}, \underline{x}]]\left[\underline{\mathbf{c}}^{-1}, \underline{x}^{-1}\right]$ des séries de Laurent formelles en $\left(\underline{x}=x-x_{0}, \underline{\mathbf{c}}=\mathbf{c}-\mathbf{c}_{0}\right)$ (les dérivations sont $\left.\partial / \partial \underline{x}, \partial / \partial \underline{c}_{i}\right)$. On considère $\mathcal{L} \mid\left[x_{0}\right]$ comme un sous-corps aux dérivations partielles de $\mathbb{C}[[\underline{\mathbf{c}}, \underline{x}]]\left[\underline{\mathbf{c}}^{-1}, \underline{x}^{-1}\right]$.

On complète ${ }^{135} \mathcal{L} \mid\left[x_{0}\right]$ (en $\mathbf{c}_{0}$ pour la topologie $\underline{\mathbf{c}}$-adique) en :

$$
\widehat{\mathcal{L}} \mid\left[\mathbf{c}_{0}, x_{0}\right] \subset \mathbb{C}[[\underline{\mathbf{c}}, \underline{x}]]\left[\underline{\mathbf{c}}^{-1}, \underline{x}^{-1}\right] .
$$


On voudrait définir $G$-Galois $(L / K)\left[\mathbf{c}_{0}, x_{0}\right]$ par $\left.\operatorname{Aut}\left(\widehat{\mathcal{L}} \mid\left[\mathbf{c}_{0}, x_{0}\right]\right) / K(\mathbf{c})\right)$, mais il y a plusieurs problèmes ${ }^{136}$. Umemura les surmonte et construit ([U16, Proposition 4.7]) un foncteur en groupes :

$$
\left.\operatorname{Inf}-a u t\left(\widehat{\mathcal{L}} \mid\left[\mathbf{c}_{0}, x_{0}\right]\right) / \widehat{K(\underline{\mathbf{c}})}\right):(\operatorname{Alg} / \mathbb{C}) \rightarrow(G r p),
$$

le groupe de Galois infinitésimal de l'extension différentielle $L / K$ avec les données initiales $\left(\mathbf{c}_{0}, x_{0}\right)$. C'est un foncteur de Lie-Ritt sur le corps des constantes $\mathbb{C}$, qu'il note $\operatorname{Inf}-\operatorname{gal}(L / K)\left[\mathbf{c}_{0}, x_{0}\right]$.

Ce foncteur dépend des conditions initiales. Hiroshi Umemura le compare au foncteur Inf-gal $(L / K)$ (que j'ai décrit plus haut), qui est intrinsèque mais défini sur le « gros » $\operatorname{corps} L^{\natural}$ :

"In fact, in the definition of $\operatorname{Inf-gal}(L / K)\left[\mathbf{c}_{0}, x_{0}\right]$, we choose a point $x_{0}$ that is called a $\mathbb{C}$-valued point in the language of algebraic geometry and consider the Taylor expansion around the reference point $x_{0} \in \mathbb{A}_{1}(\mathbb{C})=$ $\operatorname{Spec} \mathbb{C}[x]$. Let us carry it out at the generic point. This is done by the universal Taylor expansion, which we are going to explain." "

Ainsi le morphisme de Taylor universel sert à passer au point générique (au sens algébrique). La construction de $\mathbf{I n f - g a l}(L / K)\left[\mathbf{c}_{0}, x_{0}\right]$ reste dans la ligne des idées de Drach et Vessiot. En revanche, le passage au point générique algébrique est une idée complètement nouvelle d'Umemura.

Dans le même article, il aborde la comparaison de sa théorie avec celle de Malgrange ([U16, p. 332]) :

"It is very natural to ask how Malgrange's Galois theory of foliations and ours of differential field extensions are related. First of all, comparison requires assumptions under which both theories work. [...] Let L/K be an ordinary differential field extension such that the field $L^{\natural}$ is finitely generated over $K^{\natural}$. Then, we have $\operatorname{Inf-gal}(L / K)\left[\mathbf{c}_{0}, x_{0}\right]$ as we introduced in Subsection 4.9. On Malgrange's theory side, we need an analytic space and a foliation on it. In his theory, a particular attention is paid to get not only a Lie algebra but also a global Lie pseudo-group. For a comparison with our theory, however, we need only Lie algebra. Hence, the question is local. »

Umemura pose alors la question suivante ([U16, Question 4, p. 332]) :

Do we have an isomorphism of Lie algebras :

$$
\operatorname{Lie}\left(\left(\operatorname{Mgal}(L / K)\left[\mathbf{c}_{0}, x_{0}\right]\right)=\operatorname{Lie}\left(\operatorname{Inf}-\operatorname{gal}(L / K)\left[\mathbf{c}_{0}, x_{0}\right]\right) .\right.
$$

Le problème est encore essentiellement ouvert. 
Umemura est revenu sur la comparaison des deux théories dans [U19] :

"We sketch a proof of equivalence of two general differential Galois theories, Malgrange's theory and ours, if the base field consists only of constants. "

En fait, il traite essentiellement un exemple (celui de l'équation différentielle paramétrée ${ }^{137}$ de la Figure 1). Il évoque aussi la question dans [U18] (pour $K=\mathbb{C}$ ). Je vais faire une brève analyse de cette note.

Soit $(L, \delta)$ un corps différentiel. On suppose $L$ de type fini sur $\mathbb{C}$. Il existe un modèle lisse $(\operatorname{Spec} A, F(\delta))=(V, F(\delta))$ de l'extension de corps $L / \mathbb{C}$; $F(\delta)$ étant un champ de vecteurs algébriques sur la variété algébrique $V$ (et $A$ l'anneau des fonctions régulières sur $V$ ). On suppose, pour simplifier, $V$ affine ${ }^{138}$.

Umemura considère le groupoïde de Lie des jets formels inversibles (noté plus haut $\widehat{J}^{*}(V)$ ). Il le note $\mathbb{J}^{0}$ et note $\mathbb{J}$ l'ensemble des jets non nécessairement inversibles. Il désigne par $\operatorname{Gal}(L / \mathbb{C})$ le groupoïde de Malgange de $(V, F(\delta))$. C'est le plus petit $D$-groupoïde sur $V$ (Umemura dit sur $V \times V)$ dont l'algèbre de Lie contient le champ de vecteurs $F(\delta)$.

Umemura écrit : "Notre définition du Groupe de Galois infinitésimal nous fournit une construction du groupoïde $\operatorname{Gal}(L / \mathbb{C})$. " Je reprends son $\operatorname{argumentation}^{139}$.

L'algèbre $A$ est étale sur $\mathbb{C}\left[z_{1}, \ldots, z_{n}\right]$ et les dérivations $\partial / \partial z_{i}$ s'étendent en des dérivations $\partial_{i}$ sur $A$. Soit $\iota: A \rightarrow A[[t]]$ le morphisme de Taylor universel. On note $\left(A^{\natural}, \delta_{i}\right)$ l'algèbre $A[[x]]$ munie des dérivations $\delta_{i}$ agissant sur les coefficients et $\mathcal{A}$ la sous-algèbre engendrée par $A^{\natural}$ et $\iota(A)$ (on l'appelle anneau d'Umemura).

On a un isomorphisme canonique d'algèbres $A \otimes_{C_{A}} A \rightarrow A^{\natural}[\iota(A)]$. On en déduit un morphisme de schémas $\operatorname{Spec} \mathcal{A} \rightarrow V \times V$ en composant des morphismes de schémas :

$\operatorname{Spec} \mathcal{A} \rightarrow \operatorname{Spec} A^{\natural}[\iota(A)] \approx \operatorname{Spec} A \otimes_{C_{A}} A \rightarrow \operatorname{Spec} A \otimes_{\mathbb{C}} A=V \times V$.

Umemura montre qu'il existe une $V \times V$ immersion fermée ${ }^{140} \operatorname{Spec} \mathcal{A} \rightarrow \mathbb{J}$. Il identifie $\operatorname{Spec} \mathcal{A}$ à son image, ce qui lui permet d'interpréter Spec $\mathcal{A} \cap J^{0} \rightarrow$ $V \times V$ comme un groupoïde, et il énonce (voir son Théorème 2, qu'il ne démontre pas) :

$\operatorname{Spec} \mathcal{A} \cap \mathbb{J}^{0} \rightarrow V \times V$

est le groupoïde de $\operatorname{Galois} \operatorname{Gal}(L / \mathbb{C})$ de l'extension $L / \mathbb{C}$.

D'après B. Malgrange [72], il faut modifier cet énoncé et l'argumentation d'Umemura $^{141}$. Il faut remplacer le groupoïde de Malgrange $\operatorname{Gal}(L / \mathbb{C})$ par 
sa partie d'ordre 0, définie par les intégrales premières. On pourrait vraisemblablement ensuite obtenir un résultat pour les ordres supérieurs à partir des prolongements du champ de vecteurs $F(\delta)$; les indications manquent à ce sujet, de même que pour la reconstitution, à l'inverse, du groupe d'Umemura à partir de $\operatorname{Gal}(L / \mathbb{C})$.

Le dernier thème que je voudrais évoquer dans cette partie est celui des relations entre la théorie d'Umemura et celle des solitons de Sato ${ }^{142}$ (dont j'ai parlé plus haut). Entre 2009 et 2011, Umemura a présenté de nombreuses conférences en France, quelques unes au Japon, une en Pologne ${ }^{143}$ et une aux $\mathrm{USA}^{144}$ sur ce sujet. Je n'ai malheureusement trouvé que peu de traces écrites de son travail ${ }^{145}$; seulement [U6, U7]. Je reproduis ici le résumé de sa conférence à Niigata en 2010 [U6] : Sato's Soliton theory is abelian.

Abstract. "Since the success of Morales-Ramis theory that LiouvilleArnold integrability is detected Galois theoretically, we encountered many non-integrable systems. It is marvelous that the principle is so effective. On the other hand, Soliton theory is one of the most remarkable theories developed in the last century. It started in the 19th century by observation of phenomena, mathematical modeling, inverse scattering method, experiments combined with computer science and finally Sato formulated mathematically the whole theory. We observe Sato's theory by our general Galois theory to conclude that Galois group of KP hierarchy is abelian. This shows that KP hierarchy is very much integrable as expected. The result is a simple Galois theoretic interpretation of Sato's great theory. "

Il y a dans [U7] quelques détails techniques. Malheureusement certaines définitions et les démonstrations sont manquantes.

Umemura s'est intéressé dans [83] (avec Morikawa) à l'application de sa théorie à l'intégrabilité des systèmes dynamiques. Leur philosophie est : "In theory of dynamical systems, we believe in general what is integrable is abelian ». Ils illustrent ce principe en rappelant les résultats du type MoralesRamis et l'appliquent à des systèmes discrets. L'idée est que si un système dynamique discret est intégrable ${ }^{146}$, alors le groupe formel associé Inf-gal est abélien. Supposons par exemple que le système est l'opération d'un élément $g$ d'un groupe algébrique $G$ sur une variété algébrique $V^{147}$. Alors le groupoïde de Malgrange est l'adhérence de Zariski $\overline{\langle g\rangle}$ du sous-groupe $\langle g\rangle$ engendré par $g$ et il est évidemment abélien. Morikawa et Umemura associent au système une extension aux différences $L / C(V)$ et montrent que Inf-gal $(L / C(V))$ est « isomorphe » à $L^{\natural} \otimes_{C}$ Lie $\overline{\langle g\rangle}$, donc abélien.

J'avais auparavant montré des résultats similaires (pour le cas différentiel) en utilisant la théorie de Malgrange : si un système hamiltonien est intégrable, alors l'algèbre de Lie de son groupoïde de Malgrange est abélienne ${ }^{148}$. J'ai 
exposé ce résultat en 1999 à un colloque ${ }^{149}$ à Kyoto et Nara organisé par Tohru Morimoto [105 $]^{150}$. Umemura participait au colloque ${ }^{151}$ et nous avions discuté de la question ${ }^{152}$, il est possible que ceci l'ait influencé.

Le système dynamique de Sato sur la grassmanienne de dimension infinie est (en un sens évident) intégrable. Ainsi le résultat annoncé par Hiroshi est un premier pas vers des énoncés similaires en dimension infinie.

Comme je l'ai évoqué plus haut, en 2006 Umemura a fait un exposé [U5] dans une conférence à Marseille-Luminy en l'honneur de B. Malgrange. Sur le premier transparent figuraient deux photos du sanctuaire Fushimi-Inaritaisha au sud de Kyoto. L'une d'une statue du renard ${ }^{153}$ et l'autre de l'un des innombrables tori rouge vermillon. Hiroshi comparait la malice du renard de la tradition shintoïste au regard galoisien sur divers objets mathématiques ${ }^{154}$. Le double thème du renard et du tori était repris tout au long des transparents pour souligner en marge par deux «idéogrammes» des points clés (cf. la Figure 1).

Umemura admirait la culture japonaise traditionnelle et se situait par sa foi dans la tradition bouddhique. Je pense que les transparents que je viens d'évoquer reflètent bien la façon dont il envisageait la recherche en mathématiques et même la vie en général. Hiroshi était un excellent mathématicien et, ce qui est plus important encore, un homme d'une bonté profonde. Comme l'a écrit Pierre Cartier « un sage nous a quittés».

\section{Poursuivre l'oeuvre de Hiroshi Umemura}

Il y a de nombreux prolongements possibles du travail d'Umemura sur les groupes de Galois de dimension infinie. Je suis incompétent sur beaucoup d'entre eux et je me limiterai à deux questions (d'ailleurs liées) qui me paraissent importantes.

Je me place dans le cadre algébrique. Il y a quatre outils pour regarder « un même objet » :

(1) Le groupe « générique » d'Umemura Inf-gal $(L / K)$.

(2) le groupe d'Umemura avec conditions initiales $\operatorname{Inf}-\operatorname{gal}(L / K)\left[\mathbf{c}_{0}, x_{0}\right]$.

(3) le $D$-groupoïde Mal de Malgrange;

(4) la limite projective des groupes de Galois différentiels (de PicardVessiot) des variationelles de tous ordres le long d'une solution (Morales-Ramis-Simó [81]).

Il faudrait expliciter de manière aussi précise que possible les relations entre ces quatre approches. J'ai évoqué ci-dessus la comparaison entre (1) 
(ou (2)) et (3) selon Umemura. G. Casale a étudié les relations entre (3) et (4) [25] et récemment Malgrange a donné des indications pour prolonger ce travail en utilisant ${ }^{155}$ [72]. Notons que pour comparer (1) (ou (2)) et (3) (ou (4)), il suffit de considérer pour (2) (ou (3)) les algèbres de Lie. Ceci suffit pour les applications aux questions d'intégrabilité : il faut décider si une algèbre de Lie est abélienne, ou au moins résoluble.

On peut aussi considérer les questions analogues pour le cas des équations aux $q$-différences.

Un autre problème intéressant est la question de la confluence ${ }^{156}$ éventuelle de Inf-gal aux $q$-différences vers Inf-gal différentiel quand $q \rightarrow 1$. On peut, plus généralement, spécialiser des familles génériquement discrètes en des cas différentiels [4]. G. Casale et D. Davy ont récemment obtenu des résultats de semi-continuité de la dimension du groupoïde de Malgrange dans ce cadre ${ }^{157}[29]$ et ils les ont appliqués au cas des Painlevé discrètes. On peut raisonnablement conjecturer que le groupe Inf-gal d'Umemura « se simplifie par spécialisation », en un sens qui reste à éclaircir. Umemura formule cette idée dans [U16, Part II, 5. Question 5, p. 333] : « Behavior of Inf-gal under specialization. It would be sufficient to express logically the following fact. If we specialize an equation, we will have more constraints so that the Galois group would be smaller. »

Dans son intervention au Congrès International des Mathématiciens à Moscou en 1966, Ellis Kolchin a présenté cinq problèmes. Le quatrième consistait en deux questions ( $L$ étant un polynôme différentiel et $G$ le groupe de Galois différentiel associé) :

(1) Étant donné $L$, déterminer $G$;

(2) Étant donné $G$, déterminer un $L$ dont c'est le groupe de Galois.

La première question est appelée le problème direct, la seconde le problème inverse. Je ne parlerai que du problème direct ${ }^{158}$. Il y a eu un certain nombre de travaux sur cette question dans les dernières années du XIX-ème siècle ${ }^{159}$, puis le problème a été oublié. E. Kolchin lui même a fait peu de calculs effectifs. L'étude du problème direct a repris vers le début des années 80 . Depuis les progrès ont été considérables (en partie grâce au calcul formel). On s'est aussi intéressé à un résultat plus faible : calculer seulement l'algèbre de Lie du groupe de Galois ${ }^{160}$. On trouvera un historique de la question dans [114] and $[115,1.5]$, et, pour les progrès les plus récents, on pourra consulter l'article [6] et sa bibliographie. Ainsi le cas linéaire est déjà assez difficile. Passons maintenant aux versions non-linéaires du problème direct.

J'ai discuté avec Malgrange des problèmes de « calculabilité » au moment de son premier article sur sa théorie de Galois. Il était très sceptique sur le 
fait que l'on puisse faire des calculs effectifs, sauf dans des cas assez triviaux (et, bien sûr, dans le cas des extensions normales de Kolchin). Il semble qu'Umemura avait plus ou moins le même pessimisme vis à vis de sa théorie (voir $[\mathrm{U} 17,9$.$] ). Je suis plus optimiste et pense qu'une voie importante de$ recherches pour l'avenir est de chercher des méthodes de calcul effectif avec les deux théories ${ }^{161}$ pour des exemples « significatifs». De ce point de vue les équations de Painlevé ${ }^{162}$ sont un « challenge » évident. Il y a un certain nombre de résultats pour le groupoïde de Malgrange, que l'on devrait pouvoir traduire en termes de groupe formel d'Umemura.

Je donne un « état de l'art» pour cette famille d'exemples : calculs de groupoïdes de Galois-Malgrange et preuves « dans le style Galoisien » de l'irréductibilité.

(1) Calcul du groupoïde de Malgrange pour PI par G. Casale [24].

(2) Preuve, par G. Casale, du fait que si le groupoïde de Malgrange est « suffisamment gros », alors l'équation a une solution générale irréductible [26].

(3) Calcul du groupoïde de Malgrange pour PVI, pour tous les paramètres, par S. Cantat et Frank Loray [17] (et application à l'irréductibilité). Ce calcul est basé sur l'étude de la « dynamique d'Iwasaki » sur la variété des caractères de PVI (une surface cubique affine) : cette dynamique est engendrée par des applications polynomiales explicitables.

(4) Preuves d'irréductibilité pour PII et PIII, par G. Casale et J.A. Weil [32], dans les cas où il existe une solution algébrique avec des tehniques à la Morales-Ramis-Simó.

(5) Calcul du groupoïde de Malgrange pour PII, PIII, PIV, PV et des valeurs générales des paramètres ${ }^{163}$, par G. Casale et D. Davy [28] (cf. aussi [29] et [39]).

(6) Tentative de calcul du groupoïde de Malgrange pour PV (non dégénéré), par M. Klimes, E. Paul et J.P. Ramis par une approche à la Cantat-Loray. La dynamique d'Iwasaki sur la variété des caractères de PVI est remplacée par une « dynamique sauvage »sur la variété des caractères (sauvage) de $\mathrm{PV}^{164}$.

Il me semble qu'en mettant en œuvre tous les outils dont on dispose on peut espérer pouvoir calculer dans un avenir pas trop lointain les groupoïdes de Malgrange et les groupes infinitésimaux d'Umemura pour toutes les équations de Painlevé. Dans [U16], Umemura pose la question : « [...] calculate the Galois group Inf-gal $\left(K\left(y, y^{\prime}\right) / K\right)$ for a general solution $y$ of the first Painlevé equation. We can ask the similar question for the other Painlevé 
equations. » Il conjecture la réponse « évidente » pour PI, mais est relativement pessimiste : "It does not seem easy to prove the conjecture. Maybe, it requires a new idea."

La longue marche vers la théorie de Galois fonctionnelle ${ }^{165}$ n'est pas terminée $^{166}$. La belle théorie d'Umemura demeurera pour le pèlerin une halte naturelle sur le chemin, comme un temple bouddhiste sur le chemin spirituel de Shikoku.

\section{Remerciements}

Je remercie : Daniel Bertrand pour ses relectures, ses informations sur sa collaboration avec Hiroshi et ses suggestions pour l'amélioration de l'article, Guy Casale pour son éclairage sur les comparaisons des théories de Malgrange et d'Umemura et ses éclaircissements sur le groupe de rationalité et le groupe spécifique de Vessiot, Kazuo Okamoto pour ses informations sur M. Hukuhara et la «préhistoire » des équations de Painlevé au Japon et ses souvenirs du « bon vieux temps » à Strasbourg, Katsunori Saito pour m'avoir transmis le rapport d'auto-évaluation d'Umemura pour le JSPS, Juan J. Morales Ruiz pour m'avoir fourni les références [U6, U7] et Yousuke Ohyama pour son aide et sa documentation pour l'écriture du pararagraphe 2,

\section{Notes}

${ }^{1}$ Écrit en collaboration avec A. Matsuoka et K. Saito [U2].

${ }^{2}$ Pour sa théorie de Galois différentielle et ses relations avec celle de Bernard Malgrange je donnerai quelques détails techniques.

${ }^{3} \mathrm{Cf}$. ci-dessous mon analyse d'un passage de la « lettre testament » d'Évariste Galois [46].

${ }^{4}$ Les thèmes mathématiques majeurs qui vont apparaître dans mon texte naissent avec Painlevé ou jouent un rôle important dans son oeuvre : équations de Painlevé, feuilletages holomorphes, propriété d'irréductibilité (transcendantes nouvelles), théorie de Galois différentielle non linéaire.

${ }^{5}$ Hadamard et Painlevé s'étaient rencontrés sur les bancs du lycée, puis retrouvés à l'École Normale. Ils étaient liés par de grands liens d'amitié.

${ }^{6}$ Sur ce second point, cf. [61, 3.1, p. 120], pour une version moderne.

${ }^{7} \mathrm{Il}$ s'agir bien sûr de P. Painlevé

${ }^{8}$ C'est-à-dire dépendant des conditions initiales. 
${ }^{9} \mathrm{Ni}$ « pire » : il peut apparaître des bords naturels mobiles [34].

${ }^{10}$ Ses premiers calculs ont été corrigés et complétés par son élève B. Gambier.

${ }^{11}$ Son groupe de rationalité n'est pas toujours défini.

${ }^{12}$ Il y a eu, en 1902-1903, à propos de la «preuve » de Painlevé pour PI [96] une vive controverse entre Painlevé et R. Liouville [69] (cf. [U17, p. 270]). R. Liouville était le neveu de J. Liouville, mais il n'avait pas le génie de son oncle.

${ }^{13}$ En utilisant la classification des pseudo-groupes de Lie agissant sur $\mathbb{C}^{2}$ due à Elie Cartan [21].

${ }^{14}$ [93, p. 490-496]. Selon [30] cette définition, bien que parfois critiquée, est « irréprochable».

${ }^{15}$ Une équation d'ordre $n$ est dite réductible si l'on peut exprimer une solution rationnellement, après avoir résolu successivement des équations différentielles linéaires, abéliennes (i. e. dont les solutions sont des fonctions abéliennes) ou d'ordre strictement plus petit que $n$.

${ }^{16}$ Fils de Lazarus Fuchs.

${ }^{17}$ Plus précisément des équations à 5 singularités dont une apparente.

${ }^{18}$ Introduites quelques années auparavant par G.D. Birkhoff.

${ }^{19} \mathrm{Il}$ interprète une singularité irrégulière comme un paquet de singularités régulières infiniment proches.

${ }^{20}$ C'est Raymond Gérard qui m'en avait signalé l'existence. Un peu plus tard, Hikosaburo Komatsu, en visite à Strasbourg après Moscou et voyant l'article de Garnier sur mon bureau, m'avait dit qu'il avait vu cet article sur le bureau d' I.M. Gelfand la semaine précédente.

${ }^{21}$ L'interprétation d'une singularité irrégulière comme confluence de singularités régulières apparaît dans de nombreux travaux, cf. [67, 5.2, p. 26].

${ }^{22} \mathrm{Et}$ explique mieux leur apparition dans diverses applications.

${ }^{23}$ Elle garde un sens pour les équations d'ordre arbitraire [61]. Par contre, la méthode de Painlevé ne s'étend que partiellement aux ordres supérieurs, cf. les travaux de J. Chazy.

${ }^{24}$ Aux heures noires de l'échec de l'offensive de Nivelle au chemin des dames, des muniteries, de la paix séparée à Brest-Litovsk. . .

${ }^{25}$ Président du Conseil. Plus tard, en 1921 il fonde la Ligue de la République et il participe ensuite à la création du Cartel des gauches.

${ }^{26}$ Qui avait succédé à Painlevé pour l'enseignement de la mécanique à l'École Polytechnique.

${ }^{27} \mathrm{R}$. Garnier est une exception notable, mais il a lui aussi été oublié! Par ailleurs la propriété de Painlevé est restée dans l'arsenal des physiciens et des mécaniciens [38].

${ }^{28}$ C'était un sujet très «en dehors des modes ». L'idée était de prolonger des travaux de Levelt sur les fonctions hypergéométriques. Pus tard Gérard et Levelt ont collaboré.

${ }^{29} \mathrm{G}$. Reeb était un élève de Charles Ehresmann. Ils avaient fondé la théorie des feuilletages. Cette théorie, qui apparaît ici en relation avec Painlevé, réapparaîtra plus loin dans 
mon texte : elle joue un rôle important dans la théorie de Galois différentielle de Bernard Malgrange.

${ }^{30}$ Le but de la seconde thèse était d'ouvrir l'horizon culturel du candidat. Le sujet devait donc en principe être éloigné de celui de la première thèse, ce ne fut, heureusement, pas $\mathrm{du}$ tout le cas pour les deux thèses de Gérard.

${ }^{31}$ Ce travail d'édition prit plusieurs années et Kazuo Okamoto y a collaboré pendant son séjour strasbourgeois.

32 J'étais au milieu des annes 60 doctorant à Paris. Nous croisions parfois R. Garnier à l'Institut Henri Poincaré quand il venait boire un verre à l'ocasion d'un pot de thèse et nous aurions été bien surpris d'apprendre qu'il avait fait des choses importantes en mathématiques.

${ }^{33}$ Sauf dans quelques petites écoles d'ingénieurs. Je les ai apprises en les enseignant dans une de ces écoles. . .

${ }^{34}$ Je pense qu'il y a eu, à un demi-siècle d'intervalle, une « résonance » entre la vision de Reeb et celle de Painlevé. Je cite Étienne Ghys [55] : "[Painlevé] a l'intuition de la notion de structure feuiletée et comprend qu'il est préférable de considérer les "solutions " comme des surfaces de Riemann non paramétrées qui sont en général non singulières mais dont les projections sur les axes de coordonnées présentent des singularités. " et, plus loin : "Reeb, dans la lignée de Painlevé, commence l'étude systématique des feuilletages. Il s'agit d'objets de nature dynamique où les feuilles, remplaçant les trajectoires. [...] L'exemple de base pourrait être celui des courbes intégrales d'une forme différentielle $P(x, y) \mathrm{d} x+Q(x, y) \mathrm{d} y=0$. ».

${ }^{35}$ Les alsaciens appellent intérieur le complémentaire de l'Alsace dans l'hexagone. La topologie de l'Alsace est étrange.

${ }^{36}$ Comme je l'ai raconté ailleurs [104].

${ }^{37}$ Non sans quelques réticences de ma part au début : j'étais habitué à penser en termes de monodromie!

${ }^{38} \mathrm{La}$ bibliothèque mathématique de Strasbourg est exceptionnelle.

${ }^{39}$ Il me l'a confié lors d'une rencontre au Japon.

${ }^{40}$ Je cite Okamoto : Les Leçons de Stockholm ont été réimprimées au Japon vers 1950 à l'Université de Nagoya; on a retapé toutes les pages des Leçons à la machine à écrire... Hukuhara a publié un livre en 1950 sur la théorie des équations différentielles, en japonais, où il a mentionné les équations de Painlevé et elles sont bien connues au Japon surtout par le dictionnaire des mathématiques, version japonaise, publié en 1954. Hukuhara n'a publié qu'un mémoire sur les équations de Painlevé mais il a rédigé une note magnifique écrite en Esperanto. Je l'ai publiée en collaboration avec Takano : "The proof of the Painlevé property by Masuo Hukuhara », Funkcialaj Ekvacioj (2001).

${ }^{41}$ Équations fonctionnelles. La revue est toujours publiée par Mathematical Society of Japan.

${ }^{42}$ Umemura est aussi venu à Strasbourg en 1975-76 dans le cadre de la collaboration franco-japonaise.

${ }^{43}$ La première année, il était boursier du gouvenement français, comme Umemura l'avait été avant lui (un système efficace aujourd'hui disparu). La seconde, il était attaché de 
recherches au C.N.R.S., dans un cadre A.T.P. Par la suite Okamoto a souvent visité l'Université de Strasbourg : 13 fois dont l'une pour un colloque en son honneur en 2018. Sa dernière visite fut en septembre 2019.

${ }^{44}$ Avant d'arriver à Strasbourg il avait «essayé de comprendre la substance des Leçons de Stockolm ».

${ }^{45}$ Les exemples non triviaux connus à l'époque étaient les équations de Riccati et le cas elliptique [52]. Dans ces deux cas l'espace des conditions initiales est compact : respectivement $\mathbb{P}^{1}(C)$ et une courbe elliptique.

${ }^{46}$ Nous avions compris que c'était important sans savoir à quel point.

${ }^{47}$ Okamoto m'a récemment écrit : Quand j'ai annoncé le résultat à l'occasion du séminaire, tu m'avais dit de compléter les calculs pour toutes les équations de Painlevé; alors j'ai construit d'abord la variété des conditions initiales pour la sixième équation et j'ai obtenu la forme Hamiltonienne de l'équation. Je te suis reconnaissant de me donner conseil. J'avais oublié ma modeste contribution à l'histoire!

${ }^{48}$ Qui dépendra de $x$.

${ }^{49}$ Okamoto explique que, pour PI, on ne peut pas espérer une surface compacte en s'appuyant sur l'irréductibilité de PI, qu'il attribue à Painlevé. Il ne savait pas à cette époque que la preuve de Painlevé est incorrecte.

${ }^{50}$ Ces surfaces $\Sigma_{(\varepsilon)}^{(2)}$ sont des compactifications minimales de $\mathbb{C}^{2}$.

${ }^{51} \mathrm{La}$ construction en donne une compactification et la structure du « diviseur exceptionnel $\gg D$ (feuilles verticales) est importante. Il devait apparaître plus tard que, pour chaque équation de Painlevé, la variété des conditions initiales est caractérisée par une paire de systèmes de racines affines qui représentent respectivement le type de symétrie et le type de la surface [65].

52 " One of the common properties of the Painleve equations is that the space of initial values is obtained from $\mathbb{P}^{1}(\mathbb{C}) \times \mathbb{P}^{1}(\mathbb{C}),[\ldots]$ by blowing up at eight points. Therefore the configuration of the eight points, which possibly includes infinitely near points, provides with the most fundamental data of the equation ".

${ }^{53}$ On dit : «l'approche géométrique des équations de Painlevé ».

${ }^{54}$ Où ils montraient que la fonction de corrélation du modèle d'Ising satisfait à la troisième équation de Painlevé.

${ }^{55}$ Un lien entre les déformations isomonodromiques et la théorie quantique des champs.

${ }^{56}$ Les fonctions $\tau$ sont les fonctions génératrices pour les hamiltoniens des flots isomonodromiques (ou iso-irréguliers). Dans les applications ce sont les fonctions $\tau$, plutôt que les transcendantes de Painlevé, qui apparaissent naturellement.

${ }^{57} \mathrm{Il}$ donne deux interprétations de la dérivée logarithmique $\sigma$ de $\tau$ et montre l'identité « $\sigma=\sigma »$.

${ }^{58} \mathrm{C}$ 'est une grassmanienne en dimension infinie.

${ }^{59}$ Bien sûr il y a ailleurs beaucoup de recherches intéressantes et d'autres techniques sont développées. On peut citer, par exemple, les travaux de l'école russe, cf. le livre [48] et 
sa bibliographie, et divers travaux récents en relation avec les champs conformes, cf. [70] et sa bibliographie.

${ }^{60}$ Pierre Cartier avait quitté Strasbourg pour le C.N.R.S. Nous nous sommes croisés à Strasbourg où j'ai été nommé professeur en septembre 1971, sur le poste de Cartier ou celui de Gabriel, je ne sais plus...

${ }^{61}$ Cf. $[\mathrm{U} 12, \S 4$, p. 372] : " Thanks to theory of schemes we can avoid all the difficulties..."

${ }^{62}$ On trouvera des détails dans [71].

${ }^{63}$ Deux volumes de rédactions d'exposés ont paru dans la série Springer Lecture Notes, édités par Gérard et moi : numéros 712 et 1015.

${ }^{64}$ Inspirée, d'une part, par les invariants découverts par Gérard et Levelt à Blancherupt un soir devant quelques verres de vin d'Alsace et d'autre part, par un travail de H. Komatsu [104].

${ }^{65}$ Sans doute à la suite de l'article de Nicholas M. Katz de 1982 [66] où la version intrinsèque du groupe de Picard-Vessiot (un groupe algébrique sur le corps différentiel de base) joue un rôle crucial.

${ }^{66} \mathrm{~J}$ 'ai raconté aileurs [104] dans quelles circonstances.

${ }^{67} \mathrm{Ma}$ conférence au premier colloque franco-japonais de 1985 était sur ce sujet : sur mon théorème de densité [103].

${ }^{68}$ Pour PI, G. Casale a donné une démonstration de ce type en utilisant la théorie de Malgrange [24, 26].

${ }^{69}$ Cf. par exemple [U16, Part I, 2].

${ }^{70}$ Selon Hermann Weyl [120, p. 138] : " This letter, if judged by the novelty and profundity of ideas it contains, is perhaps the most substancial piece of writing in the whole litterature of mankind ».

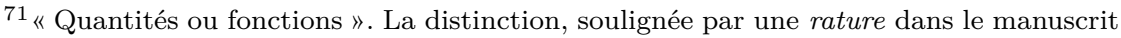
original qui est à la bibliothèque de l'Institut de France (j'ai vérifié cette rature sur le manuscrit), est importante : dans le premier cas on peut penser à des théories de Galois de nombres transcendants (comme les périodes, les zéros de certaines fonctions transcendantes), comme le pensent Y. André [3,4] et R. Pérez-Marco [15], dans le second à des théories de Galois différentielles, comme le pensaient Sophus Lie [68], Jules Drach [43] et peut-être Émile Picard. Je cite R. Pérez-Marco : «After reading it over and over, some words started to pop out and dominated the meaning. In the photographic reproduction one can see a crossing out at this point. It is written there "des quantités ou quantités fonctions transcendantes" with the second word "quantités" crossed out [...] All of this reveal that something important is communicated in these words. "

${ }^{72}$ L'autre est le principe du tiers exclu : « tertium non datur ».

${ }^{73}$ Les théories de Galois différentielles, en particulier celles d'Umemura et de Malgrange, sont des exemples de la théorie de l'ambiguïté selon Birkhoff : cf. [11, p. 45]. Les théories de la relativité de Galilée et Einstein en sont d'autres ainsi que les théories modernes des particules élémentaires (groupes de jauges) et les théories de Galois motiviques, les motifs pouvant être vus comme des sortes de "particules élémentaires» [4]. Hermann Weyl, par exemple, interprète la théorie de Galois classique comme une théorie de la relativité : 
"[Galois'] theory may be described as the algebraic relativity theory for a finite set of numbers which are given as the roots of an algebraic equation » (cf. also [120, p. 138]). Dans les débuts d'exposés d'Umemura, il y avait souvent des analogies avec des idées exprimées par H. Weyl dans son livre sur la symétrie [120] (convergence de pensées ou influence, je ne sais). Je reproduis les deux principes de Birkhoff. Ils méritent d'être mieux connus. Je les trouve assez visionnaires, bien qu'ils semblent n'avoir intéressé personne.

"Principle of sufficient reason. If there appears in any theory $T$ a set of ambiguously determined (i.e. symmetrically entering) variables, then these variables can themselves be determined only to the extent allowed by the corresponding group G. Consequently any problem concerning these variables which has a uniquely determined solution, must itself be formulated so as to be unchanged by the operations of the group $G$ (i.e. must involve the variables symmetrically). »

"Heuristic conjecture. The final form of any scientific theory $T$ is : (1) based on a few simple postulates; and (2) contains an extensive ambiguity, associated symmetry, and underlying group $G$, in such wise that, if the language and laws of the theory of groups be taken for granted, the whole theory $T$ appears as nearly self-evident in virtue of the above Principle.

Il faudrait bien sûr aujourd'hui remplacer « groupe » par 《 groupoïde » et noter que les « objets » de la théorie $\mathrm{T}$ sont des représentations du groupe d'ambiguïté $G$.

Je pense que la théorie de l'ambiguïté est un vaste courant de pensée qui, avant

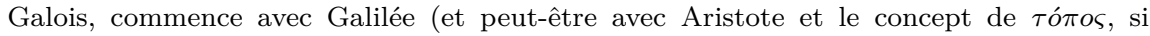
l'on en croit Hermann Weyl) et se poursuit avec Leibniz. Après Galois, il y a, outre les développements mathématiques, A. Einstein, H. Weyl [120, 121] et les théories de jauge basées sur des groupes de jauge, comme $S U(3) \times S U(2) \times U(1)$ pour le modèle standard, qui sont évidemment des « groupes d'ambiguïté ». H. Weyl écrit, dans [121, Chapter 5, p. 121] : "Aristotle already designed position (topos) as a relation of one body to the bodies of its surrounding».

${ }^{74}$ Celle de Malgrange, dont je parlerai plus loin, est une théorie géométrique basée sur les notions de feuilletage et de champ de vecteurs.

${ }^{75}$ Il suppose seulement que le corps $L$ est finiment engendré sur $K$.

${ }^{76}$ Le morphisme de Taylor peut s'interpréter comme une dérivation itérée au sens de F. Heiderich $[59,60]$. Cette dernière notion garde un sens en caractéristique positive. Pour le cas discret il y a un analogue, le morphisme d'Euler universel [82].

${ }^{77}$ Pour la théorie de Galois différentielle dans le cas paramétré, cf. [33]. Cette illustration de sa théorie est détaillée dans [U19, 5. Example 5.1, p. 447]. Cf. aussi [U17].

${ }^{78}$ Malgrange écrit dans [77], « [...] équation différentielle (ou feuilletage) au-dessus d'un feuilletage; or ce type de situation n'est pas considérée ici. Il serait nécessaire de combler cette lacune. Incidemment, ce serait aussi nécessaire pour comprendre dans quels cas de la présente théorie on a une correspondance de Galois, correspondance qui existe dans les théories de tous ces auteurs. " Notons aussi que, dans le cas linéaire, le groupe de Galois dit générique, ou intrinsèque, ou à la Katz (cf. la note 79), ne voit pas non plus la correspondance de Galois.

${ }^{79}$ Le groupe de Galois différentiel intrinsèque est une forme tordue du groupe de Galois différentiel standard, définie sur le corps différentiel de base. Dans le cadre tannakien, on le définit en termes de foncteur fibre d'oubli et de constructions $[1,8,9]$. 
${ }^{80} \mathrm{Ce}$ point de vue permet de mieux comprendre les relations entre l'approche d'Umemura et celles de Drach et Vessiot.

${ }^{81}$ Cf. le texte de Philippe Di Francesco dans [38].

${ }^{82} \mathrm{Je}$ cite [65] : "In the efforts for finding a unified framework for the Painlevé type equations, Sakai proposed a class of second order discrete Painlevé equations arising from Cremona transformations of rational surfaces obtained as nine-point blow-ups of $\mathbb{P}^{2}[107]$. Those rational surfaces are regarded as the spaces of initial values for discrete and continuous Painlevé equations, and are classified into 22 cases according to the configuration of nine points. The master equation of those Painlevé equations, the elliptic Painlevé equation, [...]»

${ }^{83}$ Fils de Kenji Nishioka et de Kumiko Nishioka.

${ }^{84}$ Pour d'autres approches, cf. [31] et le projet décrit dans [29].

${ }^{85}$ Mais Fahim, Levelt et van der Put ont montré qu'il est préférable et plus naturel d'utiliser des anneaux differentiels simples.

${ }^{86}$ Dans [41] les auteurs utilisent une approche dynamique (que l'on peut rapprocher de [56] et [83]) : "Thanks to our comparison results, we are able to compare the MalgrangeGranier D-groupoid to the parametrized Galois group of Hardouin-Singer. "

${ }^{87}$ On considère un anneau aux différences $(R, \sigma)$. \̀ $a \in R$ on associe $u_{a}: \mathbb{N} \rightarrow R^{\natural}$ défini par $n \mapsto \sigma^{n}(a)$. Si l'on munit l'anneau des applications $F\left(\mathbb{N}, R^{\natural}\right)$ du shift évident $\Sigma$, le morphisme d'Euler universel est le morphisme injectif d'anneaux aux différences $\iota_{R}:(R, \sigma) \rightarrow\left(F\left(\mathbb{N}, R^{\natural}\right), \Sigma\right)$ défini par $a \mapsto u_{a}$.

${ }^{88}$ Les théories d'Umemura et Morikawa sont en caractéristique nulle.

${ }^{89} \mathrm{D}$ 'après nos derniers échanges, j'ai l'impression qu'il voyait ce travail comme un testament mathématique.

${ }^{90} \mathrm{Je}$ me suis longtemps demandé si c'était possible sans jamais trouver d'exemple. Dans toutes les situations considérées par Yves André dans [2], il n'apparaît jamais de groupe quantique. F. Heiderich avait suggéré cette direction [59].

${ }^{91}$ Umemura avait au préalable écrit, en collaboration avec K. Saito, deux articles sur ce thème [U3, U4].

${ }^{92}$ Un groupe d'ambiguïté. De même, le pauvre algébriste ne peut observer que la violation des contraintes algébriques et le groupe correspondant est le groupe de Galois classique. Le pauvre physicien ne peut observer dans son laboratoire que la violation des lois de la physique : par exemple, les passagers de la nef de Galilée ne peuvent pas voir si elle bouge. Si l'observateur est mieux outillé, le groupe diminue. S'il est omniscient, le groupe est trivial.

${ }^{93}$ Une version « universelle».

${ }^{94}$ Les physiciens ont bien souvent un meilleur goût. Citons, par exemple, R. Conte [38], A. Ramani, B. Grammaticos [100], B. Julia, O. Lisovyy [70].

${ }^{95}$ Où ils avaient chacun fait un exposé.

${ }^{96}$ Je cite D. Bertrand : "Je venais de terminer mon article avec $W$. Zudilin On the transcendence degree of the differential field generated by Siegel modular functions, paru 
plus tard au J. reine angew. Math 554 (2003), 47-68. Pendant mon séjour à Nagoya, nous avons travaillé sur le groupe de Galois des équations différentielles algébriques correspondantes (au moins en dimension 1, à l'aide des équations schwarziennes, d'ordre 3), mais sans vraiment aboutir. " Pour une avancée remarquable sur cette question, voir [14, 30].

${ }^{97}$ Sur une fellowship de la JSPS.

${ }^{98}$ Avec les réserves habituelles sur la définition de cet objet par Drach.

${ }^{99}$ En appendice à l'article [U15], il y a un extraît d'une lettre de Malgrange à Bertrand. Malgrange reprend la question en utilisant sa version du groupe de Galois différentiel.

${ }^{100}$ Elle était à Strasbourg durant les séjours de Hiroshi et ils avaient noué une vive amitié. Elle est restée en contact avec lui et son épouse Noriko jusqu'à la fin et elle était présente à la cérémonie d'adieux à Hiroshi à Nagoya.

${ }^{101}$ Umemura avait fait un exposé à son « Séminaire Darboux », au Centre de Physique Théorique de l'E.N.S., en Avril 2015 : Toward quantization of Galois theory.

102 Par exemple la conférence Taniguchi Complex analytic theory of differential equations organisée à la villa Kyuzeso, à Katata, au bord du lac Biwa, en août 1987, par K. Okamoto.

${ }^{103}$ C'était la première thèse sur la théorie de Malgrange.

${ }^{104}$ Avec une bourse JSPS.

${ }^{105}$ Organisée par Juan Morales.

${ }^{106}$ La ville du traité, près de Valladolid.

${ }^{107}$ Perez-Marco explore depuis plusieurs années le monde qu'il appelle transalgébrique [12, 15] et a introduit pour celà tout un outillage nouveau (log-Riemann surfaces), s'écartant $\mathrm{du}$ "formalisme moderne » (cf. note 166). Sa vision est dans le droit fil de celles d'Euler, Riemann et Galois : "After careful readings, it became clear to the author of theses lines that in his last year, Galois had been trying to develop a "Galois theory" for roots of transcendental functions, and also had worked on the similar problem for functions. "

${ }^{108}$ En un sens à préciser [12].

${ }^{109}$ J'ai rencontré Hiroshi pour la première fois à Strasbourg en 1975. C'est le début de notre amitié, mais ce n'est que lors de son séjour suivant, en 1984, que nos trajectoires mathématiques se sont rencontrées, avec des échanges sur l'algèbre différentielle. Ensuite, et avant ma visite à Nagoya, nous nous sommes rencontrés plusieurs fois au Japon, en particulier à la conférence Taniguchi en 1987, voir la note 102.

${ }^{110}$ L'une pour le colloque anniversaire pour ses 60 ans

${ }^{111}$ À l'occasion de la conférence franco-japonaise Recent developments in differential equations in the complex domain organisée au RIMS par Viktoria Heu et Yousuke Ohyama, 29 ans après la première conférence à Strasbourg.

${ }^{112}$ Cette comparaison est claire pour le cas fortement normal et facile pour quelques cas particuliers. Le cas général nécessite encore un effort.

${ }^{113}$ Cette question est assez délicate et chaque mot compte. Je ne saurais faire une meilleure description. J'ai juste souligné quelques termes importants, fait une légère retouche et ajouté des notes. 
${ }^{114}$ Considérons par exemple une équation $U^{\prime}=A U$ où $U$ est une matrice inversible. on peut regarder une action $\grave{a}$ gauche : les transformations de jauge qui conservent la connexion $U \mapsto P U$ avec $P^{\prime}=[A, P]$. On peut aussi considérer une action $\grave{a}$ droite, celle de GL des constantes. Les deux actions agissent simplement transitivement sur les solutions et elles commutent. «Picard-Vessiot » (le groupe de rationalité) est un sousgroupe de l'action à droite, mais " Malgrange » (le groupe spécifique) est un sous-groupe de l'action à gauche. Pour le cas non linéaire, selon l'interprétation de G. Casale, Vessiot montre que si l'on choisit une petite boite de flot les deux objets sont isomorphes.

${ }^{115}$ Sur ces deux notions, cf. aussi $[18,6$, p. 5] : «M. Vessiot apporta des idées nouvelles à sa théorie en introduisant à côté du groupe de rationalité ce qu'il appelle le groupe spécifique, ce qui rend presque intuitive la solution du problème posé par M. Drach. Il y a isomorphisme entre les deux groupes.".

${ }^{116}$ Le groupe spécifique est le plus petit groupe rationnel contenant l'équation comme sous-groupe infinistésimal.

117 À la Picard-Vessiot-Kolchin.

${ }^{118}$ Les premiers articles de Malgrange sont écrits dans le cadre analytique. Dans la mesure où je m'intéresse dans ce texte aux rapprochements avec la théorie d'Umemura et au cas des équations de Painlevé je me limiterai essentiellement au cadre algébrique adopté par Malgrange dans [77]

${ }^{119}$ Cette construction est aussi chez B. Malgrange [77, 2.3] qui dit qu'elle a été obtenue indépendamment par P. Cartier et O. Gabber.

${ }^{120}$ Le plus petit sous-groupe algébrique de $\operatorname{GL}(n ; \mathbb{C})$ contenant $\operatorname{Im} \rho$.

${ }^{121}$ Qui apparaît chez Schlesinger.

${ }^{122} \mathrm{Il}$ y a une autre méthode utilisant les tores exponentiels et les multiplicateurs de Stokes et mon théorème de densité [101]. La comparaison entre les deux procédés, triviale pour $y^{\prime}=y$, n'est pas claire en général, même pour l'équation d'Airy $y^{\prime \prime}+x y=0$.

${ }^{123}$ Umemura l'appelle groupoïde de Poincaré.

${ }^{124}$ En définissant convenablement l'extension de Picard-Vessiot.

${ }^{125}$ On peut dire que la théorie de Galois classique est « ce que l'algèbre voit des racines ».

${ }^{126}$ C'est une variété analytique de dimension infinie. Ce groupoïde a été plus tard utilisé par Umemura dans [U19, 4, p. 6].

${ }^{127}$ Pour les deux objets $a, b \in V$, tout $\varphi:(V, a) \rightarrow(V, b)$ analytique inversible est un morphisme.

${ }^{128}$ Cette présentation est un écho du travail original de Galois et de la théorie différentielle linéaire : $\operatorname{Iso}(\cdot)$ associe à un corps le groupe qui le conserve et $\operatorname{Inv}(\cdot)$ associe à un groupe son corps d'invariants; $\operatorname{Iso}(\operatorname{Inv}(\cdot))$ est une «fermeture ».

${ }^{129}$ C'est un fibré principal. Par exemple, si $V=A^{1}$, alors :

$$
R_{k} V=A^{1} \times \mathrm{GL}_{1} \times A^{k-1}, \quad \mathbb{C}\left[R_{k} V\right]=\mathbb{C}\left[r, r^{1}, 1 / r^{1}, r^{2}, \ldots, r_{k}\right]
$$

et le groupe de structure est $\Gamma_{k}^{1}$ : la variété algébrique $\mathrm{GL}_{1} \times A^{k-1}$ avec une loi tordue.

${ }^{130}$ Les dérivations $\partial / \partial x_{i}$ induisent sur les $\mathbb{C}\left[R_{k} V\right]$ les dérivations de Cartan $D_{i}: \mathbb{C}\left[R_{k} V\right] \rightarrow$ $\mathbb{C}\left[R_{k+1} V\right]$. 
${ }^{131}$ Attention : les $\mathbb{C}\left[R_{k} V\right]$ et les $F_{k}$ ne sont pas des corps différentiels.

${ }^{132} \mathrm{Il}$ est solution d'un système d'ordre $k$ d'équations aux dérivées partielles.

${ }^{133} \mathrm{Ce}$ cas contient les équations de Painlevé.

${ }^{134}$ Comme Umemura, j'ignore les problèmes de singularités éventuelles.

${ }^{135}$ La solution $\left(z_{i}(\mathbf{c}, x)\right) \in \mathcal{L} \mid\left[x_{0}\right]$ est identifiée à son image $\underline{z}(\underline{\mathbf{c}}, \underline{x})$ dans $\mathbb{C}[[\underline{\mathbf{c}}, \underline{x}]]\left[\underline{\mathbf{c}}^{-1}, \underline{x}^{-1}\right] ;$ le corps aux dérivées partielles $\widehat{\mathcal{L}} \mid\left[\mathbf{c}_{0}, x_{0}\right]$ est engendré sur $K(\mathbf{c})$ par les $z_{i}(\underline{\mathbf{c}}, \underline{x})$.

${ }^{136}$ Extensions d'algèbres au lieu de corps, problèmes de composition d'expressions formelles.

${ }^{137}$ Hiroshi tente également de comparer sa théorie à la théorie de Picard-Vessiot paramétrée de Cassidy-Singer. Il revient de façon plus approfondie sur cette comparaison dans $[\mathrm{U} 17,7]$. On retrouve le contenu de la Figure 1 dans l'Example 7.1.

${ }^{138}$ Sinon on fait, comme Umemura, les calculs dans des cartes affines et on recolle.

${ }^{139} \mathrm{Et}$ je la commenterai dans la note 141.

${ }^{140}$ Il utilise la structure de $A \otimes_{\mathbb{C}} A$-algèbre de $\mathcal{A}$ et les dérivations $\partial_{i}$, prolongées à $\mathcal{A}$.

${ }^{141}$ Malgrange revient sur l'isomorphisme annoncé par Umemura $: \mathcal{A} \approx A \otimes_{C_{A}} A$. L'énoncé similaire où l'on remplace $A$ par le corps $L$ est vrai, mais c'est plus délicat pour l'anneau. L'anneau d'Umemura $\mathcal{A}$, engendré par $A$ et $\exp (t F(\delta)) A$, est un quotient de $A \otimes_{\mathbb{C}} A$ par un idéal $I$. Malgrange montre que $I$ est l'idéal qui définit la partie de degré 0 du groupoïde $\operatorname{Gal}(L / \mathbb{C})$.

${ }^{142}$ Plus généralement, Umemura s'est intéressé aux relations entre sa théorie et d'autres EDP comme l'équation de la chaleur.

${ }^{143} \mathrm{Au}$ centre de conférences de Bedlewo en 2010.

${ }^{144}$ À Houston en 2011 [U7].

${ }^{145}$ D'après Yousuke Ohyama et Katsunori Saito il n'y en pas d'autre, cf. le rapport d'Umemura au JSPS en mai 2011 : https://kaken.nii.ac.jp/file/KAKENHIPROJECT-20540041/20540041hyoka.pdf. Les textes [U6, U7] m'ont été transmis par Juan J. Morales Ruiz. Hiroshi et Juan avait découvert, lors de la conférence de Niigata, qu'ils tentaient tous les deux de mettre en œuvre une approche des solitons par les théories de Galois différentielles. L'une des motivations de Morales est une note de Drach [45].

${ }^{146} \mathrm{Ils}$ se placent dans un cadre algébrique.

${ }^{147}$ En caractéristique nulle.

${ }^{148}$ C'est une variante de la théorie de Morales-Ramis. En fait, on a un résultat plus précis : l'algèbre de Lie de la partie d'ordre 0 du groupoïde de Malgrange est abélienne.

${ }^{149} \mathrm{~A}$ cette époque on connaissait la théorie de Malgrange, qui n'avait pas encore été publiée.

${ }^{150}$ L'exposé n'a pas été rédigé, on trouvera la démonstration dans [81, Theorem 6]. Cf. aussi [75] et [22, 5.4, p. 79].

${ }^{151}$ Ainsi que Malgrange. 
${ }^{152}$ En décembre 1999, les rapports entre les théories de Malgrange et d'Umemura n'étaient pas du tout clairs. Ma mémoire est imprécise, mais nous avons pu penser que, dans le cas algébrique, le groupe d'Umemura d'un système hamiltonien intégrable est abélien. Les notes 148 et 141 permettent de voir que l'algèbre de Lie du groupoïde $\operatorname{Spec} \mathcal{A} \cap \mathbb{J}^{0} \rightarrow V \times V$ est abélienne.

${ }^{153}$ Une représentation de la déesse (ou plutôt Kami) Inari.

${ }^{154}$ Mon analogue occidental de la malice du renard serait la version de Birkhoff du principe de raison suffisante dont j'ai parlé plus haut, même si l'on n'imagine guère Leibniz en renard.

$155[72]$ : «Soit $X$ un champ de vecteurs sur $V$. L'avantage d'utiliser (1) est la " fonctorialité " : soit $W$ une sous-variété de $V$ stable par $X$, si l'on a un fibré sur $V$ muni d'une $X$-connexion, on aura encore une $X$-connexion au-dessus de $W$ et l'algèbre d'Umemura de $V$ s'enverra surjectivement sur celle de $W$, d'où une injection des groupes de Galois correspondants. » Ceci s'applique en particulier au cas de [81] où la solution est algébrique.

${ }^{156} \mathrm{Il}$ y a des résultats pour les théories linéaires [2, 113]. En physique, apparaissnt des déformations d'objets différentiels en objets aux $q$-diffférences (par exemple en théorie des champs conformes) avec $q=e^{2 i \pi \hbar}$, donc «très voisin de 1 ».

157 "The general opinion is that the dynamical behaviour of $\mathrm{Mal}_{s}$ for a particular value of $s \in S$ must be simpler that the behaviour on the generic fiber. "

${ }^{158}$ Je me limite au cas du corps de base $\mathbb{C}(x)$.

${ }^{159}$ A. Boulanger, M.F. Marotte, J.F.Th. Pépin.

${ }^{160}$ C'est suffisant pour nombre d'applications.

${ }^{161}$ Et leurs versions discrètes.

${ }^{162}$ Et leurs versions discrètes.

${ }^{163}$ Hors d'une union dénombrable de sous-variétés fermées.

${ }^{164}$ On considère les $\mathrm{PJ}(\mathrm{J} \neq \mathrm{I})$. En utilisant [13], on peut montrer qu'il y a des phénomènes de Stokes non-linéaires locaux pour PJ. Ces phénomènes de Stokes et les tores exponentiels correspondants se lisent, via l'application de Riemann-Hilbert (RH), comme des dynamiques locales sur la variété des caractères. Je conjecture que cette dynamique est rationnelle et effectivement calculable [97]. C'est démontré pour PV par Martin Klimes [67]. Je conjecture que l'image inverse par $\mathrm{RH}$ de cette dynamique est dans le groupoïde de Malgange. S'il en est ainsi on peut reprendre la méthode de Cantat-Loray [17].

${ }^{165}$ Et, rêvons, vers une unification avec les théories de Galois cosmiques [3, 4, 19, 20, $35,36,37]$, les théories motiviques, les relativités... Notons, par exemple, que, après renormalisation (cf. le groupe de Galois cosmique de Connes-Marcolli), les coefficients des séries perturbatives (formelles Gevrey) sont des périodes; le groupe de Galois, pour l'instant mythique, des périodes [3,4] agit donc sur ces séries. (Cet exemple prolonge une idée de P. Cartier [19, p. 25].)

166 Citons Alain Connes à propos de Galois [35] : « En fait on peut arguer que sa théorie de l'ambiguïté, fruit de ses pensées mathématiques, est comme un animal sauvage qui n'a toujours pas été vraiment capturé par le formalisme moderne. " 


\section{Références à des travaux de H. Umemura}

[U1] D. Bertrand \& H. Umemura, « On the definitions of the Painlevé equations », RIMS Kokyuroku 1296 (2002), p. 29-34.

[U2] A. Masuoka, K. Saito \& H. Umemura, « Toward quantization of Galois theory », Ann. Fac. Sci. Toulouse, Math. 29 (2020), n 5, p. 1319-1431.

[U3] K. Saito \& H. Umemura, « Quantization of Galois theory. Examples and observations », https://arxiv.org/abs/1212.3392v1, 2012.

[U4] - "Can we quantize Galois theory? ", to appear in Proceedings of Various aspects of the Painlevé equations, https://arxiv.org/abs/1306.3660v1, 2013.

[U5] H. Umemura, "Foundation of general differential Galois theory », Marseille, November 27, 2006, lecture slides.

[U6] - "Sato's Soliton theory is abelian », Abstract of a lecture at the 4th Workshop on Hamiltonian Systems and Related Topics, Niigata University, October 14-15, 2010.

[U7] - "Soliton theory is abelian », Lecture at ACA 2011, The 17th International Conferences on Applications of Computer Algebra, Houston, June 28, 2011. http://divizio.perso.math.cnrs.fr/UMEMURA/Umemura_Soliton_theory_ is_abelian_2011.pdf.

[U8] - « Birational automorphism groups and differential equations », in Équations différentielles dans le champ complexe, Vol. II (Strasbourg, 1985), Publ. Inst. Rech. Math. Av., vol. 1985, Univ. Louis Pasteur, 1988, p. 119-227.

[U9] — , "On the irreducibility of the first differential equation of Painlevé », in Algebraic geometry and commutative algebra. In honor of Masayoshi Nagata. Volume II, Konokuniya Company Ltd., 1988, p. 771-789.

[U10] - « Second proof of the irreducibility of the first differential equation of Painlevé », Nagoya Math. J. 117 (1990), p. 125-171.

[U11] - " The Painlevé equation and classical functions », Sugaku 57 (1995), nº 4, p. 341-359, English translation in Sugaku Expo. 11 (1998), no. 1, p. 77-100.

[U12] - «Differential Galois theory of infinite dimension », Nagoya Math. J. 144 (1996), p. 59-135.

[U13] - « Galois theory of algebraic and differential equations », Nagoya Math. J. 144 (1996), p. 1-58.

[U14] — , « Lie-Drach-Vessiot theory, infinite-dimensional differential Galois theory », in CR-geometry and overdetermined systems, Advanced Studies in Pure Mathematics, vol. 25, Kinokuniya Company, 1997, p. 364-385.

[U15] — , « Monodromy preserving deformation and differential Galois group. I », in Analyse complexe, systèmes dynamiques, sommabilité des séries divergentes et théories galoisiennes. I. Volume en l'honneur de Jean-Pierre Ramis, Astérisque, vol. 296, Société Mathématique de France, 2004, p. 253-269.

[U16] - " Galois theory and Painlevé equations », in Asymptotic theories and Painlevé equations, Séminaires et Congrès, vol. 14, Société Mathématique de France, 2006, p. 29-339.

[U17] — , «nvitation to Galois theory », in Differential equations and quantum groups, IRMA Lectures in Mathematics and Theoretical Physics, vol. 9, European Mathematical Society, 2007, p. 269-289.

[U18] — - «Sur l'équivalence des théories de Galois différentielles générales », C. $R$. Math. Acad. Sci. Paris 346 (2008), no 21-22, p. 1155-1158.

[U19] - « On the definition of the Galois groupoid », in Équations différentielles et singularités. En l'honneur de J. M. Aroca, Astérisque, vol. 323, Société Mathématique de France, 2009, p. 441-452. 


\section{Bibliographie}

[1] Y. ANDRÉ, "Quatre descriptions des groupes de Galois différentiels », in Séminaire d'algèbre Paul Dubreil et Marie-Paule Malliavin, Lecture Notes in Mathematics, vol. 1296, Springer, 1987, p. 28-41.

[2] — , "Différentielles non commutatives et théories de Galois différentielle ou aux différences », Ann. Sci. Éc. Norm. Supér. 34 (2001), n 5, p. 685-739.

[3] , " Galois Theory, Motives and Transcendental Numbers », https://arxiv . org/abs/0805.2569v1, 2008.

[4] — , "Idées galoisiennes ", in Histoires de mathématiques (Journées mathématiques X-UPS 2011), Éditions de l'École polytechnique, 2012, p. 1-16.

[5] T. Archibald, «Differential Equations and Algebraic Transcendents : French Efforts at the Creation of a Galois Theory of Differential Equations 1880-1910», Rev. Hist. Math. 17 (2011), no 2, p. 373-401.

[6] M. Barkatou, T. Cluzeau, J.-A. Weil \& L. Di Vizio, Computing the Lie Algebra of the Differential Galois Group of a Linear Differential System, ACM Press, 2016.

[7] D. Bertrand, "Groupes algébriques linéaires et théorie de Galois différentielle », Cours de 3ème cycle à l'Université Pierre et Marie Curie (Paris 6), notes de cours rédigées par René Lardon, 1985-86.

[8] - « Review of Lectures on Differential Galois theory by A. Magid », Bull. Am. Math. Soc. 3 (1996), p. 289-294.

[9] — , "Remarks on the intrinsic inverse problem », in Differential Galois theory, Banach Center Publications, vol. 58, Polish Academy of Sciences, 2002, p. 27-35.

[10] G. D. Birkhoff, «The Generalized Riemann Problem for Linear Differential Equations and the Allied Problems for Linear Difference and $q$-Difference Equations », Proc. Am. Acad. 49 (1913), no 9, p. 521-568.

[11] — - "The principle of sufficient reason », Rice Inst. Pamphlet 28 (1941), $\mathrm{n}^{\circ}$ 1, p. 24-50, reproduced in Collected Mathematical Papers, vol. 3, American Mathematical Society, 1950.

[12] K. Biswas \& R. Perez-Marco, «Log-Riemann Surfaces », https://arxiv.org/abs/ 1512.03776v1, 2015.

[13] A. Bittmann, « Doubly-resonant saddle-nodes in $\left(\mathbb{C}^{3}, 0\right)$ and the fixed singularity at infinity in Painlevé equations : analytic classification », Ann. Inst. Fourier 68 (2018), $\mathrm{n}^{\circ} 4$, p. $1715-1830$.

[14] D. Blázquez-Sanz, G. Casale, J. Freitag \& J. Nagloo, « Some Functional Transcendence Results aroud the Schwarzian Differential Equation», Ann. Fac. Sci. Toulouse, Math. 29 (2020), nº 5, p. 1265-1300.

[15] D. Burch, N. Jones, E. Muñoz-García \& R. PÉrez-Marco, « Transalgebraic numbers », Preliminary unfinished version, UCLA (2002), unpublished. (Communication personnelle.).

[16] F. Cano, F. Loray, J. J. Morales-Ruiz, P. Sad \& M. Spivakovsky (éds.), Équations différentielles et singularités. En l'honneur de J. M. Aroca, Astérisque, vol. 323, Société Mathématique de France, 2009.

[17] S. Cantat \& F. Loray, « Dynamics on Character Varieties and Malgrange irreducibility of Painlevé VI equation», Ann. Inst. Fourier 59 (2009), n 7, p. 2927-2978.

[18] É. Cartan, «L'oeuvre scientifique de M. Ernest Vessiot», Bull. Soc. Math. Fr. 75 (1947), p. 1-8.

[19] P. Cartier, « Notes sur l'histoire et la philosophie des mathématiques $\mathrm{V}$ : le problème de l'espace », https://www. academia.edu/534424. 
[20] - «A mad day's work : from Grothendieck to Connes and Kontsevich. The evolution of concepts of space and symmetry », Bull. Am. Math. Soc. 38 (2001), no 4 , p. 389-408.

[21] — - "Groupoïdes de Lie et leurs algébroïdes », in Séminaire Bourbaki. Volume 2007/2008. Exposés 982-996, Astérisque, vol. 326, Société Mathématique de France, 2008, p. 2007-2008.

[22] G. Casale, « Sur le groupoïde de Galois d'un feuilletage », Thèse, Université Paul Sabatier (Toulouse III), 2004.

[23] - " Enveloppe galoisienne d'une application rationnelle de $\mathbb{P}^{1} »$, Publ. Mat., Barc. 50 (2006), no 1, p. 191-202.

[24] - " Le groupoide de Galois de $P_{1}$ et son irréductibilité », Comment. Math. Helv. 83 (2008), no 3, p. 471-519.

[25] — , "Morales-Ramis Theorems via Malgrange pseudogroup », Ann. Inst. Fourier 59 (2009), no 7, p. 2593-2610.

[26] — - « Une preuve galoisienne de l'irréductibilité au sens de Nishioka-Umemura de la première équation de Painlevé », in Équations différentielles et singularités. En l'honneur de J. M. Aroca, Astérisque, vol. 323, Société Mathématique de France, 2009, p. 83-100.

[27] — - "An introduction to Malgrange Pseudogroup », in Théories de Galois et arithmétique des équations différentielles, Séminaires et Congrès, vol. 23, Société Mathématique de France, 2011.

[28] G. Casale \& D. Davy, «Specialisation of the Galois groupoid of a vector field ", https://arxiv.org/abs/2004.09122, 2004.

[29] - "Galois groupoid and confluence of difference equations », https://arxiv . org/abs/2006.02675v1, 2006.

[30] G. Casale, J. Freitag \& J. Nagloo, « Ax-Lindemann-Weierstrass with derivatives and the genus 0 Fuchsian groups », Ann. Math. 192 (2020), n 3, p. 721-765.

[31] G. Casale \& J. Roques, « Dynamics of rational symplectic mappings and difference Galois theory ", Int. Math. Res. Not. 2008 (2008), article no. rnn103 (23 pages).

[32] G. Casale \& J.-A. Weil, " Galoisian methods for testing irreducibility of order two nonlinear differential equations », Pac. J. Math. 297 (2018), no 2, p. 299-337.

[33] P. Cassidy \& M. F. Singer, « Galois theory of parametrized differential equations and linear differential algebraic group », in Differential equations and quantum groups, IRMA Lectures in Mathematics and Theoretical Physics, vol. 9, European Mathematical Society, 2007, p. 113-155.

[34] J. Chazy, «Sur les équations différentielles dont l'intégrale générale possède une coupure essentielle mobile », C. R. Math. Acad. Sci. Paris (1910), p. 456-458.

[35] A. Connes, «Exposé de clôture, Galois », www.alainconnes.org/docs/ slidesgaloisihp1.

[36] — , "Renormalisation et ambiguïté galoisienne 》, in Analyse complexe, systèmes dynamiques, sommabilité des séries divergentes et théories galoisiennes. I. Volume en l'honneur de Jean-Pierre Ramis, Astérisque, vol. 296, Société Mathématique de France, 2004, p. 113-143.

[37] A. Connes \& M. Marcolli, Noncommutative Geometry, Quantum Fields and Motives, Colloquium Publications, vol. 55, American Mathematical Society, 2008.

[38] R. Conte (éd.), The Painlevé property one century later, CRM Series in Mathematical Physics, Springer, 2012.

[39] D. DAVY, «Spécialisation du pseudo-groupe de Malgrange et irréductibilité », Thèse, Université Rennes 1, 2013.

[40] P. Deligne, Équations différentielles à points singuliers réguliers, Lecture Notes in Mathematics, vol. 163, Springer, 1970. 
[41] L. Di Vizio \& C. Hardouin, «Intrinsic approach to Galois theory of $q$-difference equations ", to appear in Mem. Am. Math. Soc., https://arxiv.org/abs/1002. 4839v5, 2019.

[42] L. Di Vizio, J.-P. Ramis, J. Sauloy \& C. Zhang, «Équations aux $q$-différences », Gaz. Math., Soc. Math. Fr. 96 (2003), p. 20-49.

[43] J. Drach, «Essai sur une théorie générale de l'intégration et sur la classification des transcendantes », Ann. Sci. Éc. Norm. Supér. (1898), p. 243-384.

[44] — - "Sur les équations différentielles du premier ordre et du premier degré », $C$. R. Math. Acad. Sci. Paris 158 (1914), p. 926-929.

[45] - « Détermination des cas de réduction de l'équation différentielle $\mathrm{d}^{2} y / \mathrm{d} x^{2}=$ $[\phi(x)+h] y »$, C. R. Math. Acad. Sci. Paris 168 (1919), p. 47-50.

[46] G. Évariste, «Lettre à Auguste Chevalier, 29 mai 1832 », Revue encyclopédique 55, p. 568-576, Le manuscrit original de cette lettre est à la bibliothèque de l'Institut de France, Quai de Conti, Paris.

[47] D. Foata, « Raymond Gérard (1932-2000)», Gaz. Math., Soc. Math. Fr. 84 (2000), p. $1932-2000$.

[48] A. S. Fokas, A. R. Its, A. A. Kapaev \& V. Y. Novokshenov, Painlevé transcendents. The Riemann-Hilbert approach, Mathematical Surveys and Monographs, vol. 128, American Mathematical Society, 2006.

[49] R. Fuchs, «Sur quelques équations différentielles linéaires du second ordre », $C . R$. Math. Acad. Sci. Paris 141 (1906), p. 55-558.

[50] — - « Über lineare homogene Differentialgleichungen zweiter Ordnung mit drei im Endlichen gelegenen wesentlich singulären Stellen », Math. Ann. 63 (1907), p. 301321.

[51] R. Garnier, «Sur les singularités irrégulières des équations différentielles linéaires », Journ. de Math. 2 (1919), p. 99-200.

[52] R. Gerard, « Geometric theory of differential equations in the complex domain », in Complex Anal. Appl., int. Summer Course Trieste 1975, Vol. II, 1976, p. 269-308.

[53] R. GÉrard \& A. Sec, «Feuilletages de Painlevé», Bull. Soc. Math. Fr. 100 (1972), p. $47-72$.

[54] R. GÉrard \& H. Tahara, "Singular Nonlinear Partial Differential Equations », in Structure of solutions of differential equations, Aspects of Mathematics, World Scientific, 1996, p. 135-150.

[55] É. GHys, « Les systèmes dynamiques holomorphes », in Dynamique et géométrie complexes, Panoramas et Synthèses, vol. 8, Société Mathématique de France, 1999, p. $1-10$.

[56] A. Granier, "A Galois D-groupoid for $q$-difference equations », Ann. Inst. Fourier 61 (2011), no 4, p. 1493-1516.

[57] J. Hadamard, «L'oeuvre scientifique de Paul Painlevé », Revue de Metaphys. et de Morale 41 (1934), no 3, p. 289-325.

[58] C. Hardouin \& M. F. Singer, « Differential Galois theory of linear difference equations », Math. Ann. 342 (2008), no 2, p. 333-377, erratum in ibid. 350 (2011), no. 1, p. 243-244.

[59] F. Heiderich, « Galois theory of module fields », Thèse, Universitat de Barcelona, Departament d'Álgebra i Geometria, 2010.

[60] - "Introduction to the Galois theory of Artinian simple module algebras », in Geometric and differential Galois theories, Séminaires et Congrès, vol. 27, Société Mathématique de France, 2013, p. 69-92.

[61] K. Imasaki, H. Kimura, S. Shimomura \& M. Yoshida, From Gauss to Painlevé, A Modern Theory of Special Functions, Aspects of Mathematics, vol. E16, Vieweg \& Sohn, 1991. 
[62] M. Jimbo \& T. Miwa, « Deformation of Linear Ordinary Differential Equations. I », Proc. Japan Acad. 56 (1980), p. 143-148.

[63] M. Jimbo, T. Miwa \& K. Ueno, « Monodromy preserving deformation of linear ordinary differential equations with rational coefficients. I : General theory and $\tau$ function », Physica D 2 (1981), nº 2, p. 306-352.

[64] J.-P. Jounnolou, Équations de Pfaff algébriques, Lecture Notes in Mathematics, vol. 708, Springer, 1979.

[65] K. Kajiwara, M. Noumi \& Y. Yamada, « Geometric Aspects of Painlevé quations », https://arxiv.org/abs/1509.08186v8, 2017.

[66] N. M. KATZ, «A conjecture in the arithmetic theory of differential equations », Bull. Soc. Math. Fr. 110 (1982), p. 203-239.

[67] M. Klimes, « The wild monodromy of the Painlevé $V$ equation and its action on the wild character variety : an approach of confluence », https://arxiv.org/abs/1609. 05185v3, 2019.

[68] S. Lie, "Influence de Galois sur le développement des mathématiques », in Le centenaire de l'École normale (1795-1895), Éditions Rue d'Ulm, 1895, p. 481-489.

[69] R. Liouville, "Sur les transcendantes uniformes, définies par des équations différentielles du second ordre », C. R. Math. Acad. Sci. Paris 135 (1902), p. 952-954.

[70] O. Lisovyy, «Équations de Painlevé et théorie des champs conformes », 2014, Mémoire d'Habilitation, Physique mathématique, Université de Tours, https://hal. archives-ouvertes.fr/tel-01426173.

[71] M. Loday-Richaud, "Souvenirs strasbourgeois », in Analyse complexe, systèmes dynamiques, sommabilité des séries divergentes et théories galoisiennes. I. Volume en l'honneur de Jean-Pierre Ramis, Astérisque, vol. 296, Société Mathématique de France, 2004, p. 33-42.

[72] B. Malgrange, « Courriel à Casale, Guy du 4 septembre 2020 ».

[73] - "Remarques sur les points singuliers des équations différentielles », C. R. Math. Acad. Sci. Paris 273 (1970), p. 1136-1137.

[74] — - « Le groupoïde de Galois d'un feuilletage », in Essays on geometry and related topics, Monographies de l'Enseignement Mathématique, vol. 2, L'Enseignement Mathématique, 2001, p. 465-501.

[75] — " On nonlinear Galois differential theory », Chin. Ann. Math., Ser. B 23 (2002), no 2 , p. 219-226.

[76] — - "Déformations isomonodromiques, forme de Liouville, fonction $\tau$ », Ann. Inst. Fourier 54 (2004), n 5, p. 1371-1392.

[77] — « Pseudogroupes de Lie et théorie de Galois différentielle », IHES/M/10/11, https://hal.archives-ouvertes.fr/hal-00469778, 2010.

[78] J. Martinet \& J.-P. Ramis, " Problèmes de modules pour des équations différentielles non linéaires du premier ordre », Publ. Math., Inst. Hautes Étud. Sci. 55 (1982), p. 63-164.

[79] — - "Classification analytique des équations différentielles non linéaires résonnantes du premier ordre », Ann. Sci. Éc. Norm. Supér. 16 (1983), p. 571-62.

[80] B. M. McCoy, C. A. Tracy \& T. T. Wu, « Painlevé Equations of the Third Kind », J. Math. Phys. 18 (1977), p. 1058-1092.

[81] J. J. Morales-Ruiz, J.-P. Ramis \& C. Simó, « Integrability of Hamiltonian systems and differential Galois groups of higher variational equations », Ann. Sci. Éc. Norm. Supér. 40 (2007), nº 6, p. 845-884.

[82] S. Morikawa, « On a general difference Galois theory. I », Ann. Inst. Fourier 59 (2009), no 7 , p. 2709-2732. 
[83] S. Morikawa \& H. Umemura, « On a general difference Galois theory. II », Ann. Inst. Fourier 59 (2009), n 7 , p. 2733-2771.

[84] K. NishiokA, « A note on the transcendency of Painlevé's first transcendent », Nagoya Math. J. 109 (1988), p. 63-67.

[85] S. Nishioka, « Decomposable extensions of difference fields », Funkc. Ekvacioj 53 (2010), nº 3, p. 489-501.

[86] Y. Ohyama, « Soliton Theory and Mikio Sato », in Sato's collected works, Springer, to appear.

[87] K. Окамото, "Sur les feuilletages associés aux équations du second ordre à points critiques fixes de P. Painlevé, Espaces des conditions initiales », Jpn. J. Math. 5 (1979), $\mathrm{n}^{\circ} 1$, p. 1-79.

[88] - " On the $\tau$-function of the Painlevé equations », Physica D 2 (1981), nº 3, p. $525-535$.

[89] - " Studies on the Painlevé Equations, I. Sixth Painlevé equation $P_{V I}$ », Ann. Mat. Pura Appl. 146 (1986), p. 337-381.

[90] — - "The Hamiltonians associated to the Painlevé equations », in The Painlevé property one century later, CRM Series in Mathematical Physics, Springer, 1999, p. 735-787.

[91] K. Окамото \& Y. Онуама, « Mathematical works of Hiroshi Umemura », Ann. Fac. Sci. Toulouse, Math. 29 (2020), n ${ }^{\circ}$ 5, p. 1053-1062.

[92] Ø. Ore, « Theory of Non-Commutative Polynomials », Ann. Math. 34 (1933), nº 3 , p. $480-508$.

[93] P. PAInlevé, Leçons sur la théorie analytique des équations différentielles, professées à Stockholm (1895), Hermann, 1897 (Oeuvres I, pp. 199-807).

[94] - Analyse des Travaux Scientifiques Jusqu'en 1900, Blanchard, 1900 (Oeuvres I, p. 75-196).

[95] — , "Mémoire sur les équations différentielles dont l'intégrale générale est uniforme », Bull. Soc. Math. Fr. 28 (1900), p. 201-261.

[96] - « Démonstration de l'irréductibilité absolue de l'équation $y_{x} x=6 y^{2}+x$ », C. R. Math. Acad. Sci. Paris 135 (1902), p. 641-647.

[97] E. Paul \& J.-P. Ramis, " Dynamics on Wild Character Varieties », SIGMA, Symmetry Integrability Geom. Methods Appl. 11 (2015), article no. 068 (21 pages).

[98] É. PiCARD, «Sur les équations différentielles et les groupes algébriques de transformations », Ann. Fac. Sci. Toulouse, Math. 1 (1887), p. 1-15.

[99] M. van Der Put \& M. F. Singer, Galois Theory of Difference Equations, Lecture Notes in Mathematics, vol. 1666, Springer, 1997.

[100] A. Ramani, B. Grammaticos \& J. Hietarinta, « Discrete versions of the Painlevé equations », Phys. Rev. Lett. 67 (1991), nº 14, p. 1829-1832.

[101] J.-P. Ramis, "Complete Integrability of Hamiltonian Systems and Differential Galois groups », exposé (non rédigé) au Colloque Lie Groups, Geometric Structures and differential equations - One hundred years after Lie, Sophus -, organisé par Tohru Morimoto 1999 au RIMS Kyoto University et à la Nara Women's University.

[102] — - "Dévissage Gevrey », in Journees singulieres de Dijon, 12-16 juin 1978, Astérisque, vol. 59-60, Société Mathématique de France, 1978, p. 173-204.

[103] - «Filtration Gevrey sur le groupe de Picard-Vessiot d'une équation différentielle irrégulière », in Singularités irrégulières, correspondance et documents, Documents Mathématiques, vol. 5, Société Mathématique de France, 2007, p. 129-153.

[104] — - "Singularités irrégulières : des estimations Gevrey aux théories de Galois, un itinéraire naturel », in Singularités irrégulières, correspondance et documents, Documents Mathématiques, vol. 5, Société Mathématique de France, 2007. 
[105] — - « Poincaré et les développements asymptotiques (Première partie) », Gaz. Math., Soc. Math. Fr. 133 (2012), p. 33-72.

[106] M.-H. Saito, T. Takebe \& H. Terajima, « Okamoto-Painlevé pairs and Painlevé equations », https://arxiv.org/abs/math/0006026, 2000.

[107] H. SAKAI, "Rational surfaces associated with affine root systems and geometry of the Painlevé equations », Commun. Math. Phys. 220 (2001), nº 1, p. 165-229.

[108] M. SATO, « Soliton equations as dynamical systems on infinite-dimensional Grassmann manifolds », RIMS Kokyuroku 439 (1981), p. 30-46.

[109] M. Sato, T. Miwa \& M. Jimbo, « Holonomic Quantum Fields. I. », Publ. Res. Inst. Math. Sci. 14 (1978), p. 223-267.

[110] — - "Holonomic Quantum Fields. II. The Riemann-Hilbert Problem », Publ. Res. Inst. Math. Sci. 15 (1979), p. 201-278.

[111] - " Aspects of holonomic quantum fields. Isomonodromic deformation and Ising model », in Complex analysis, microlocal calculus and relativistic quantum theory, Lecture Notes in Physics, vol. 126, Springer, 1980, p. 42-491.

[112] M. Sato \& Y. SATo, "Soliton equations as dynamical systems on infinitedimensional Grassmann manifolds ", in Nonlinear partial differential equations in applied science (Tokyo, 1982), North-Holland Mathematics Studies, vol. 81, NorthHolland, 1983, p. 25-271.

[113] J. SAUlOY, « Systèmes aux $q$-différences singuliers réguliers : classification, matrice de connexion et monodromie », Ann. Inst. Fourier 50 (2000), nº 4, p. 1021-1071.

[114] M. F. Singer, « Direct and Inverse Problems in Differential Galois Theory », Introduction to Selected works of Ellis Kolchin, American Mathematical Society, 1998.

[115] _ - "Introduction to the Galois Theory of Linear Differential Equations », in Algebraic theory of differential equations, London Mathematical Society Lecture Note Series, vol. 357, London Mathematical Society, 2009, p. 1-82.

[116] E. Vessiot, "Sur la théorie de Galois et ses diverses généralisations », Ann. Sci. Éc. Norm. Supér. 21 (1904), p. 9-85.

[117] — - "Sur la réductibilité des équations aux dérivées partielles non linéaires du premier ordre à une fonction inconnue », Ann. de l'Éc. Norm. (3) 32 (1915), p. 137160.

[118] — - "Sur une théorie générale de la réductibilité des équations et systèmes d'équations finies ou différentielles », Ann. Sci. Éc. Norm. Supér. 63 (1946), p. 1-22.

[119] W. Wasow, Asymptotic Expansions for Ordinary Differential equations, Dover Publications, 1965 .

[120] H. WeyL, Symmetry, Princeton University Press, 1952.

[121] - Mind and nature, Selected Writings on Philosophy, Mathematics and Physics, Princeton University Press, 2009.

[122] T. T. Wu, B. M. McCoy, C. A. Tracy \& E. Barouch, « Spin-spin correlation functions for the two-dimensional Ising model : Exact theory in the scaling region », Phys. Rev. B 13 (1976), nº 1, p. 316-374. 